\title{
XMM-Newton observations of IGR J18410-0535: the ingestion of a clump by a supergiant fast X-ray transient
}

\author{
E. Bozzo ${ }^{1}$, A. Giunta ${ }^{2}$, G. Cusumano ${ }^{3}$, C. Ferrigno ${ }^{1}$, R. Walter $^{1}$, S. Campana ${ }^{4}$, M. Falanga ${ }^{5}$, G. Israel ${ }^{2}$, and L. Stella ${ }^{2}$
}

\author{
${ }^{1}$ ISDC Data Center for Astrophysics, University of Geneva Chemin d'Écogia 16, 1290 Versoix, Switzerland \\ e-mail: enrico.bozzo@unige.ch \\ 2 INAF - Osservatorio Astronomico di Roma, via Frascati 33, 00044 Rome, Italy \\ 3 INAF - Istituto di Astrofisica Spaziale e Fisica Cosmica di Palermo, via U. La Malfa 153, 90146 Palermo, Italy \\ 4 INAF - Osservatorio Astronomico di Brera, via Emilio Bianchi 46, 23807 Merate (LC), Italy \\ 5 International Space Science Institute (ISSI), Hallerstrasse 6, 3012 Bern, Switzerland
}

Received 15 February 2011 / Accepted 24 May 2011

\section{ABSTRACT}

\begin{abstract}
Context. IGR J18410-0535 is a supergiant fast X-ray transients. This subclass of supergiant X-ray binaries typically undergoes fewhour-long outbursts reaching luminosities of $10^{36}-10^{37} \mathrm{erg} \mathrm{s}^{-1}$, the occurrence of which has been ascribed to the combined effect of the intense magnetic field and rotation of the compact object hosted in them and/or the presence of dense structures ("clumps") in the wind of their supergiant companion.

Aims. IGR J18410-0535 was observed for $45 \mathrm{ks}$ by XMM-Newton as part of a program designed to study the quiescent emission of supergiant fast X-ray transients and clarify the origin of their peculiar X-ray variability.

Methods. We carried out an in-depth spectral and timing analysis of these XMM-Newton data.

Results. IGR J18410-0535 underwent a bright X-ray flare that started about $5 \mathrm{ks}$ after the beginning of the observation and lasted for $\sim 15 \mathrm{ks}$. Thanks to the capabilities of the instruments on-board XMM-Newton, the whole event could be followed in great detail. The results of our analysis provide strong convincing evidence that the flare was produced by the accretion of matter from a massive clump onto the compact object hosted in this system.

Conclusions. By assuming that the clump is spherical and moves at the same velocity as the homogeneous stellar wind, we estimate a mass and radius of $M_{\mathrm{cl}} \simeq 1.4 \times 10^{22} \mathrm{~g}$ and $R_{\mathrm{cl}} \simeq 8 \times 10^{11} \mathrm{~cm}$. These are in qualitative agreement with values expected from theoretical calculations. We found no evidence of pulsations at $\sim 4.7 \mathrm{~s}$ after investigating coherent modulations in the range $3.5 \mathrm{~ms}-100 \mathrm{~s}$. A reanalysis of the archival ASCA and Swift data of IGR J18410-0535, for which these pulsations were previously detected, revealed that they were likely to be due to a statistical fluctuation and an instrumental effect, respectively.
\end{abstract}

Key words. X-rays: binaries - stars: individual: IGR J18410-0535 - stars: neutron - X-rays: stars

\section{Introduction}

IGR J18410-0535 (=AXJ1841.0-0536) is a member of the supergiant fast X-ray transients (SFXT), a subclass of supergiant $\mathrm{X}$-ray binaries (sgHMXBs) that has attracted much attention in the past few years because of their peculiar behavior in the X-ray domain (see, e.g. Walter \& Zurita Heras 2007, for an updated list of sources in this class). In contrast to previously known $\mathrm{sgH}-$ MXBs, which are nearly persistent in X-rays (see, e.g. Chaty 2010, for a review), SFXTs usually undergo few-hour long outbursts reaching luminosities of $10^{36}-10^{37} \mathrm{erg} \mathrm{s}^{-1}$, and spend most of their lifetime in quiescence, with typical luminosities of $10^{32}-10^{33} \mathrm{erg} \mathrm{s}^{-1}$ (Sidoli 2010; Bozzo et al. 2010). The presence of black-hole accretors in these sources cannot be completely ruled out. However, the properties of their X-ray spectra in outburst and quiescence and the detection of pulsations in the X-ray emission of some SFXTs, led to the conclusion that (at least) most of them should host neutron-star (NS) accretors (see e.g., Bozzo et al. 2008b, and references therein).

IGR J18410-0535 was discovered with ASCA in 1994 (Bamba et al. 2001). During the discovery observation, the source displayed two bright flares with rising times shorter than $1 \mathrm{~h}$ and separated by 0.6 days. The peak fluxes were 2.0 and $9.5 \times 10^{-11} \mathrm{erg} \mathrm{cm}^{-2} \mathrm{~s}^{-1}(2-10 \mathrm{keV})$, respectively. A timeresolved spectral analysis of the event revealed that the source
X-ray spectrum could be accurately modelled with an absorbed power-law of photon index $\Gamma=1-2$ and an absorption column density of $N_{\mathrm{H}}=(3.2-7.2) \times 10^{22} \mathrm{~cm}^{-2}$. An iron line with a centroid energy of $6.4 \mathrm{keV}$ and an equivalent width $(E W)$ of $\sim 0.2 \mathrm{keV}$ was also required by the data. During the brightest of the two flares, pulsations were reported at a period of $\sim 4.7 \mathrm{~s}$ in only the 1.9-4.9 keV energy band. The lowest X-ray flux of the source measured with ASCA was $2.0 \times 10^{-12} \mathrm{erg} \mathrm{cm}^{-2} \mathrm{~s}^{-1}$ (2-10 keV).

IGR J18410-0535 was observed with Chandra for $20 \mathrm{ks}$ on 2004 May 12 (Halpern et al. 2004). This observation captured the source with an average flux of $4.2 \times 10^{-12} \mathrm{erg} \mathrm{cm}^{-2} \mathrm{~s}^{-1}(0.5-$ $10 \mathrm{keV}$ ), and the corresponding X-ray spectrum could be described well by an absorbed power-law model with $\Gamma=1.35 \pm$ 0.30 and $N_{\mathrm{H}}=(6.1 \pm 1.0) \times 10^{22} \mathrm{~cm}^{-2}$. This observation also provided an improved position of the source at $\alpha_{\mathrm{J} 2000}=$ $18^{\mathrm{h}} 41^{\mathrm{m}} 0.54$ and $\delta_{\mathrm{J} 2000}=-05^{\circ} 35^{\prime} 46^{\prime \prime} .8$ (nominal Chandra position accuracy $0.6^{\prime \prime}$ ), and permitted the identification of the optical and infrared counterparts of the source as a B1 Ib supergiant star at an estimated distance of $3.2_{-1.5}^{+2.0} \mathrm{kpc}$ (Nespoli et al. 2008).

The source was also captured undergoing several few-hourlong outbursts with MAXI (Negoro et al. 2010) and INTEGRAL (1-7 h; Rodriguez et al. 2004; Sguera et al. 2006; Walter \& Zurita Heras 2007). The highest flux measured by INTEGRAL 
during these events was $120 \mathrm{mCrab}$ (20-80 keV; Sguera et al. 2006), corresponding to roughly $1.8 \times 10^{-9} \mathrm{erg} \mathrm{cm}^{-2} \mathrm{~s}^{-1}$. The source high energy spectrum $(20-80 \mathrm{keV})$ while in outburst could be described using a hot blackbody model (BB) with $k T=$ $8-9 \mathrm{keV}$. Outside the outbursts, the upper limit ( $1 \sigma \mathrm{c} .1$. $)$ to the average hard X-ray flux of the source was estimated to be $1 \mathrm{mCrab}$ (18-60 keV, corresponding to roughly $1.2 \times 10^{-11} \mathrm{erg} \mathrm{cm}^{-2} \mathrm{~s}^{-1}$, Filippova et al. 2004).

IGR J18410-0535 was detected a few times in outburst by Swift/BAT, and only once Swift performed a slew on the source to point it with the narrow field instrument XRT (de Pasquale et al. 2010; Romano et al. 2010a, 2011). On that occasion, the Swift XRT spectrum was closely described by an absorbed power-law model with $\Gamma=0.7_{-0.4}^{+0.5}$ and $N_{\mathrm{H}}=$ $3_{-1}^{+2} \times 10^{22} \mathrm{~cm}^{-2}$. The average $2-10 \mathrm{keV}$ unabsorbed flux was $7 \times 10^{-10} \mathrm{erg} \mathrm{cm}^{-2} \mathrm{~s}^{-1}$. No evidence for pulsations was found. IGR J18410-0535 was not detected in a high X-ray activity phase during the one-year monitoring (total net on-source exposure time $96.5 \mathrm{ks}$ ) with Swift/XRT carried out by Romano et al. (2009). In the data acquired during this monitoring, the authors identified four different states for the X-ray emission of IGR J18410-0535: the "high", "medium", "low", and "very low" states. In the high state, the source X-ray flux was $8.0 \times 10^{-11} \mathrm{erg} \mathrm{cm}^{-2} \mathrm{~s}^{-1}(2-10 \mathrm{keV})$ and the corresponding spectrum is closely described by an absorbed power-law model with $\Gamma=1.1 \pm 0.1$ and $N_{\mathrm{H}}=(2.5 \pm 0.3) \times 10^{22} \mathrm{~cm}^{-2}$. The medium state was characterized by a flux of $3.4 \times 10^{-11} \mathrm{erg} \mathrm{cm}^{-2} \mathrm{~s}^{-1}$, a power-law photon index of $1.3 \pm 0.2$, and $N_{\mathrm{H}}=(3.5 \pm 0.5) \times$ $10^{22} \mathrm{~cm}^{-2}$. For the low and very low states, the authors estimated fluxes of $1.1 \times 10^{-11} \mathrm{erg} \mathrm{cm}^{-2} \mathrm{~s}^{-1}$ and $6.0 \times 10^{-13} \mathrm{erg} \mathrm{cm}^{-2} \mathrm{~s}^{-1}$, respectively. The corresponding power-law photon indices and absorption column densities were $\Gamma=1.5 \pm 0.1,0.6 \pm 0.4$ and $N_{\mathrm{H}}=(3.5 \pm 0.5) \times 10^{22} \mathrm{~cm}^{-2},(0.6 \pm 0.4) \times 10^{22} \mathrm{~cm}^{-2}$. Sidoli et al. (2008) also reported the detection of pulsations at $\sim 4.7$ s from IGR J18410-0535 in some of the Swift/XRT data. A possible association between IGR J18410-0535 and the transient MeV EGRET source 3EGJ1837-0423 was suggested by Sguera et al. (2009).

In this paper, we report on a $45 \mathrm{ks}$ long observation of the source with XMM-Newton. This observation was obtained as part of our program aimed at studying the quiescent emission of SFXTs (see also Bozzo et al. 2010, 2009, 2008b). During the XMM-Newton observation, IGR J18410-0535 was caught undergoing a bright $\mathrm{X}$-ray flare, lasting roughly $15 \mathrm{ks}$. The source reached a maximum flux of $3.4 \times 10^{-10} \mathrm{erg} \mathrm{cm}^{-2} \mathrm{~s}^{-1}(1-10 \mathrm{keV})$ at the peak of the flare and then decayed to a very low quiescent level $\left(8.8 \times 10^{-14} \mathrm{erg} \mathrm{cm}^{-2} \mathrm{~s}^{-1}\right)$. The total dynamic range in the $\mathrm{X}$-ray flux was thus $\gtrsim 4 \times 10^{3}$. In Sect. 2 , we give the details of our data analysis and provide all the results in Sects. 2.2 and 2.3. In the XMM-Newton data, no pulsation at $4.7 \mathrm{~s}$ could be detected, and the derived upper limits were significantly tighter than the pulsation amplitude reported previously with ASCA and Swift. Motivated by these results, we also reanalyzed all the ASCA and Swift data of the source and show that the previously reported $4.7 \mathrm{~s}$ pulsations were probably due a statistical fluctuation (ASCA) and an instrumental effect (Swift), respectively (Sects. 3 and 4). Our discussion and conclusions are reported in Sects. 5 and 6.

\section{XMM-Newton data analysis}

XMM-Newton (Jansen et al. 2001) observed IGR J18410-0535 from 55270.5484 MJD to 55271.0923 MJD (exposure time $\sim 45 \mathrm{ks}$ ). The Epic-pn camera (Strüder et al. 2001) was operated

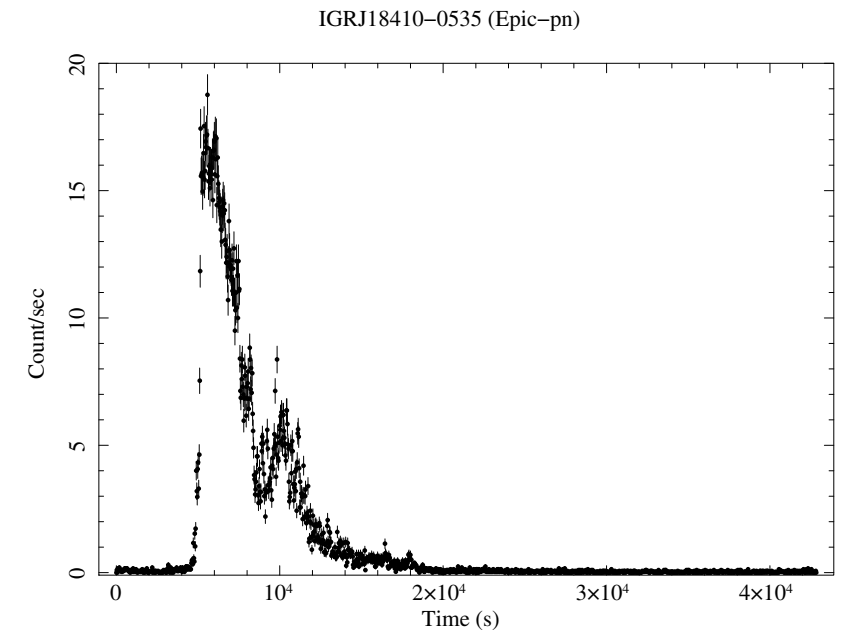

Fig. 1. XMM-Newton Epic-pn lightcurve of the observation of IGR J18410-0535 (0.3-12 keV, not corrected for background and pileup). The start time is MJD 55270.5896 (bin time $30 \mathrm{~s}$ ).

in full frame, while the Epic-MOS1 and Epic-MOS2 cameras (Turner et al. 2001) operated in small window and timing mode, respectively. This particular set-up of the instruments was chosen in order to have the capabilities required to study the source over a wide range of X-ray flux. We processed the $X M M-N e w t o n$ observation data files with the two pipelines EPPROC and EMPROC in order to produce Epic-pn and Epic-MOS cleaned event files, respectively (SAS v.10.0.1). No time intervals were found to be affected by a high background and we thus retained for the subsequent analysis the entire exposure time available for the three instruments. We used the energy range $0.15-15 \mathrm{keV}$ for the Epic-pn and $0.3-12 \mathrm{keV}$ for the two Epic-MOS cameras. IGR J18410-0535 displayed a large dynamical range in the X-ray flux during the XMM-Newton observation (see Fig. 1); therefore the source and background extraction regions were chosen in the different time intervals so as to maximize the signal-to-noise ration $(S / N)$ of the data (see next section). During the periods of greatest X-ray emission from the source, the Epic-pn data suffered significant pile-up. We accounted for this problem by using in these cases annular extraction regions for the source in which the appropriate innermost portion of the instrument point spread function (PSF) was removed $^{1}$. We checked that the removal of pile-up was effective by using the SAS tool EPATPLOT and comparing the results from the Epic-pn camera with those obtained with the EpicMOS1 and Epic-MOS2. The effect of pile-up in these cases was much reduced since the cameras were operated in small window and timing mode, respectively. Where required, we corrected Epic images to remove the out-of-time (OoT) events ${ }^{2}$ and checked that this problem did not significantly affect the spectra. All the Epic lightcurves were barycentered by using the SAS tool BARYCORR, and then corrected for instrumental vignetting, dead time, and PSF losses by using the tool EPICLCCORR. Given the lower count rate and $S / N$ of the Epic-MOS1 and Epic-MOS2 cameras compared to those obtained from the Epic-pn (especially outside the flare), the former instruments did

\footnotetext{
1 See also http://xmm.esac.esa.int/external/xmm_user_support/ documentation/uhb/index.html

2 See

http://xmm.esa.int/sas/current/documentation/threads/ EPIC_OoT.shtml
} 


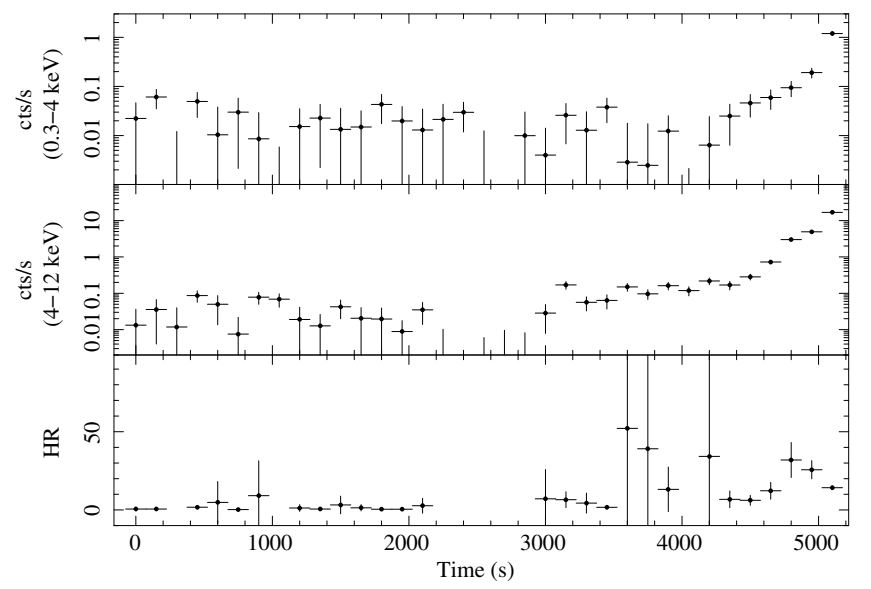

Fig. 2. Rise of the flare observed during the XMM-Newton observation of IGR J18410-0535 with the Epic-pn camera. The start time of the lightcurve is the same as that in Fig. 1, and the binning time is $150 \mathrm{~s}$. The upper panel shows the source lightcurve in the soft energy band (0.3$4 \mathrm{keV})$, the middle panel in the hard energy band (4-12 keV), and the bottom panel the hardness ratio (HR, defined as the ratio of the source count-rate in the soft to hard energy band versus time).

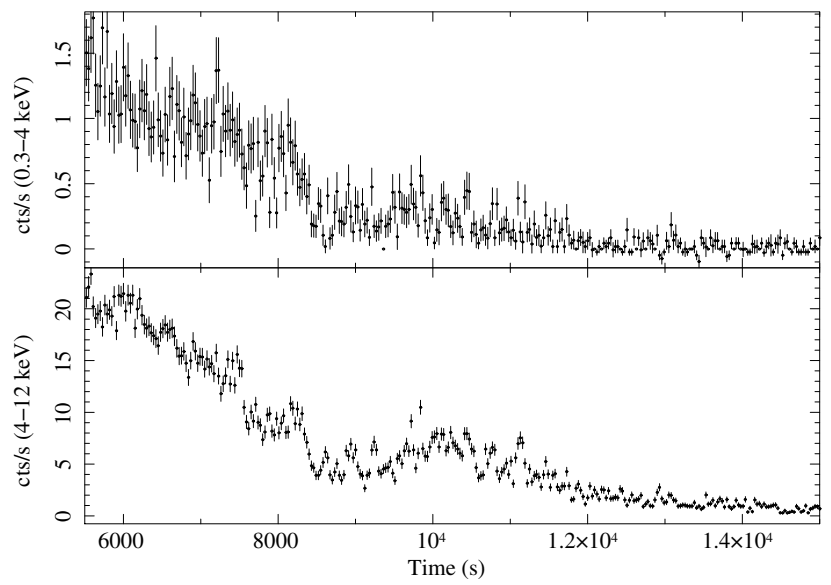

Fig. 3. Details of the Epic-pn lightcurve during the drop in count rate that occurred around $t=8500-9500 \mathrm{~s}$ in Fig. 1 ( $t=0$ would correspond to the the start time of the lightcurve in Fig. 1; time bin is $30 \mathrm{~s})$. The upper (lower) panel shows the source lightcurve in the 0.3-4 keV (4$12 \mathrm{keV}$ ) energy band.

not contribute significantly to the spectral analysis. Therefore, we report in the following sections and in Fig. 1 only the results obtained from the Epic-pn camera.

\subsection{Lightcurve analysis}

During the XMM-Newton observation, IGR J18410-0535 underwent a bright X-ray flare that started about $5 \mathrm{ks}$ after the beginning of the observation and lasted for $\sim 15 \mathrm{ks}$. The lightcurve of the entire XMM-Newton observation in the $0.3-12 \mathrm{keV}$ energy band is shown in Fig. 1.

The lowest source count-rate was recorded during the latest $20 \mathrm{ks}$ of observation at a level of $(4.0 \pm 1.9) \times 10^{-3} \mathrm{cts} \mathrm{s}^{-1}$ (estimated from a $5000 \mathrm{~s}$ binned lightcurve that had background subtracted; all uncertainties in this paper are given at $90 \%$ c.l., unless otherwise indicated). During the peak of the flare, the corresponding highest count-rate was $31.2 \pm 1.0 \mathrm{cts} \mathrm{s}^{-1}$ (determined from a $30 \mathrm{~s}$ binned lightcurve, which was background subtracted

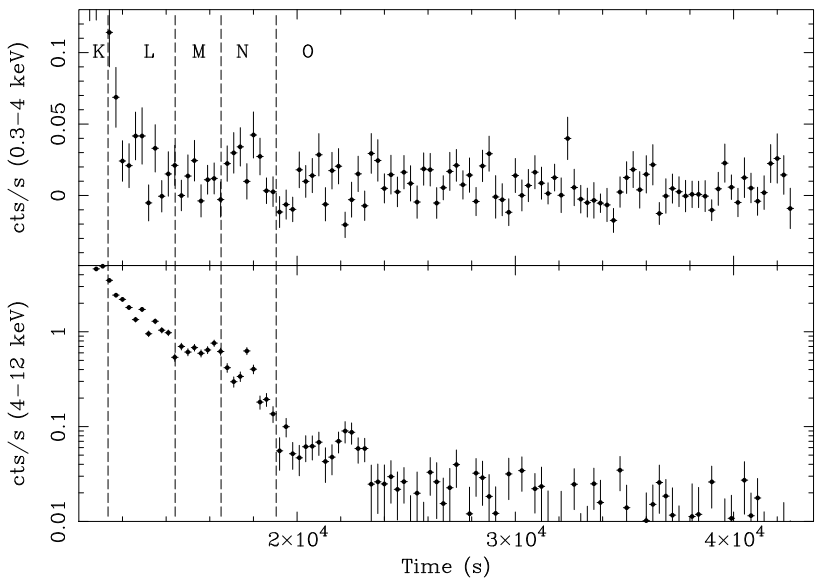

Fig. 4. Final part of the decay after the flare observed by XMM-Newton. The upper (lower) panel shows the source lightcurve in the $0.3-4 \mathrm{keV}$ (4-12 keV) energy band. We marked with vertical dashed lines the time intervals during which major changes in the source spectrum occurred (spectra K, L, M, N, O in Table 1 and Fig. 8).

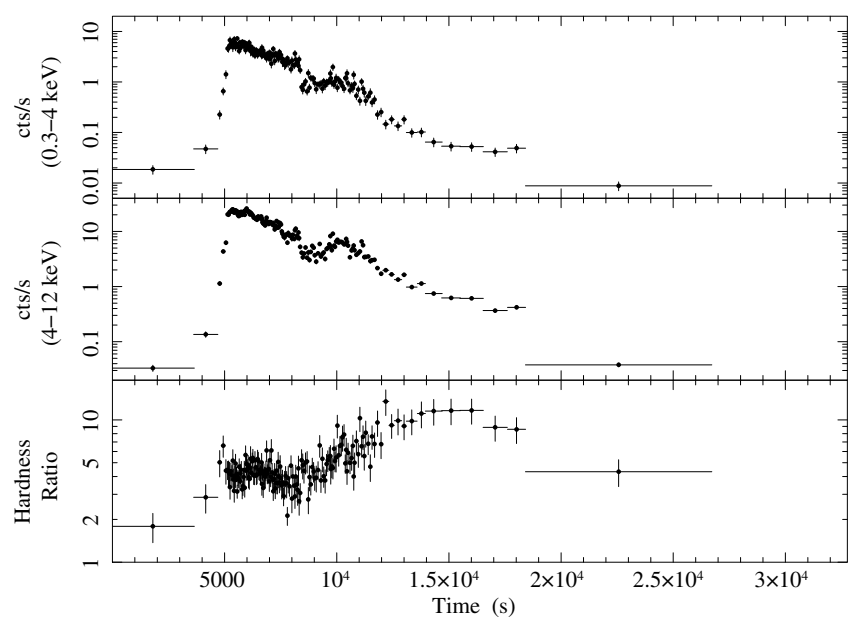

Fig. 5. The same as Fig. 1, but here we used an adaptive rebinning with $S / N=5$. The upper panel shows the source lightcurve in the $0.3-4 \mathrm{keV}$ energy band, the middle panel the source lightcurve in the 4-12 keV energy band, and the lower panel the hardness ratio versus time (defined as in Fig. 2).

and corrected for pile-up). In Fig. 2, we show an enlargement of the lightcurve during the first $5 \mathrm{ks}$ of the XMM-Newton observation. Here, a sudden rise of the source X-ray emission was recorded by the Epic-pn camera $\sim 3 \mathrm{ks}$ after the beginning of the observation. In the following $\sim 2 \mathrm{ks}$, the source reached the highest count-rate, and then began to decrease at $t \sim 6000 \mathrm{~s}$. Interestingly a drop in the source count rate was recorded around $t=8500 \mathrm{~s}$ in both the soft and hard energy band. A zoom in of this part of the lightcurve is provided in Fig. 3. A detailed lightcurve of the final part of the flare decay is shown in Fig. 4. We also show in Fig. 5 the source lightcurves in the $0.3-4 \mathrm{keV}$ and 4-12 keV energy bands, where an adaptive rebinning of the data was used in order to achieve in each time bin $S / N=5$ and estimate the corresponding source hardness ratio (HR). The same lightcurves and adaptive rebinning were used to produce the hardness ration versus intensity diagram shown in Fig. 6 (see also Bozzo et al. 2010).

From Figs. 5 and 6, we can see that the HR of the source underwent dramatic changes in time; a non-monotonic trend 


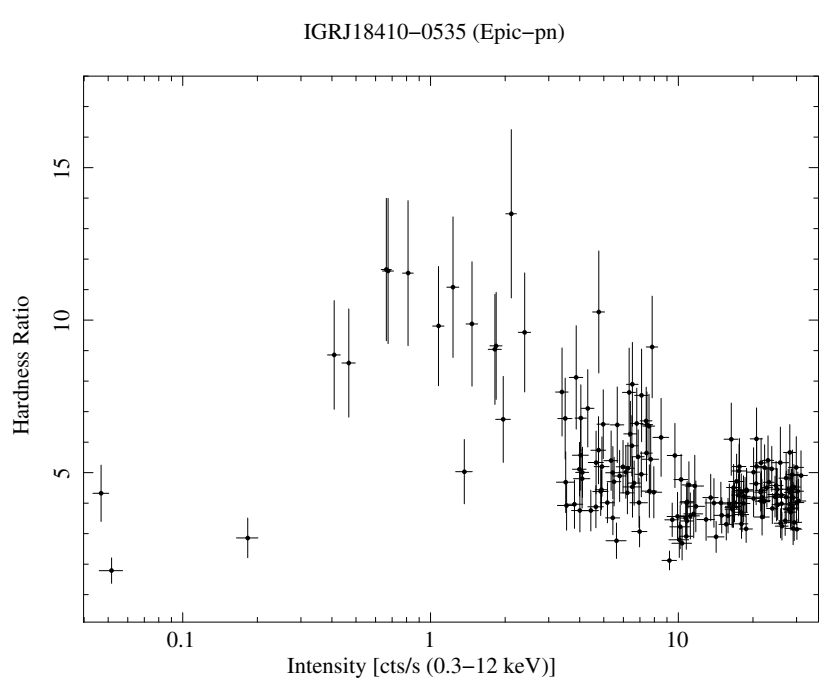

Fig. 6. Hardness-intensity diagram of IGR J18410-0535 obtained by using the XMM-Newton Epic-pn observation. The hardness ratio of the source is defined as in Fig. 2.

appears when the HR is plotted as a function of the total source intensity. Given these findings, we performed in Sect. 2.2 below a time-resolved spectral analysis of the data.

\subsection{Spectral analysis}

To search for spectral changes, we first accumulated X-ray spectra on time intervals of $\sim$ few hundreds to $\sim$ few thousands seconds (depending on the source count-rate), following the trend of the HR (see Fig. 5). Nearby time intervals were then combined in order to extract a lower number of spectra that could maximize the evidence for changes in the properties of the source $\mathrm{X}$-ray emission. Our optimal separation of the time intervals is reported in Table 1. All these spectra were rebinned to have at least 15,20 , or 25 photons per bin (depending on the source intensity) and prevent an oversampling of the energy resolution of the instruments by more than a factor of three. Spectra with lower quality statistics were rebinned to have at least 5 photons per bin and were fit using C-statistics (Cash 1979). For the spectral fits, we used an absorbed power-law model ${ }^{3}$. None of the spectra could be convincingly better fit with a blackbody (BB) or a cut-off power-law model (CUTOFFPL in XSPEC). In particular, the latter provided in most cases peculiar values of the model parameters (negative power-law photon indices and values of the high energy cut-off largely inconsistent between the different spectra). A plot of the evolution of the spectral parameters of the source in the XMM-Newton observation is provided in Fig. 7. The power-law photon index remained fairly constant up to $t \simeq 19000 \mathrm{~s}$, whereas the absorption column density underwent dramatic changes (Fig. 7). In particular, we measured a large increase (factor of $\sim 10$ ) in the absorption column density from the beginning of the observation up to the peak of the flare $\left(N_{\mathrm{H}} \sim 2 \times 10^{23} \mathrm{~cm}^{-2}\right.$ at $\left.t \simeq 5000 \mathrm{~s}\right)$. A rapid drop in the $N_{\mathrm{H}}$ then occurred to $\sim 10^{23} \mathrm{~cm}^{-2}$. At the peak of the flare, we also detected a significant iron line with an energy of $\sim 6.6 \mathrm{keV}$ and an $E W \sim 60 \mathrm{eV}$ (see Table 1). The absorption column density remained virtually constant at $\sim 10^{23} \mathrm{~cm}^{-2}$ for about $4 \mathrm{ks}$ after the source reached the highest $\mathrm{X}$-ray emission level; a rapid rise in column density then occurred until $t \simeq 19000 \mathrm{~s}$. To test the

\footnotetext{
${ }^{3}$ We used the model phabs* pow in XSPEC.
}

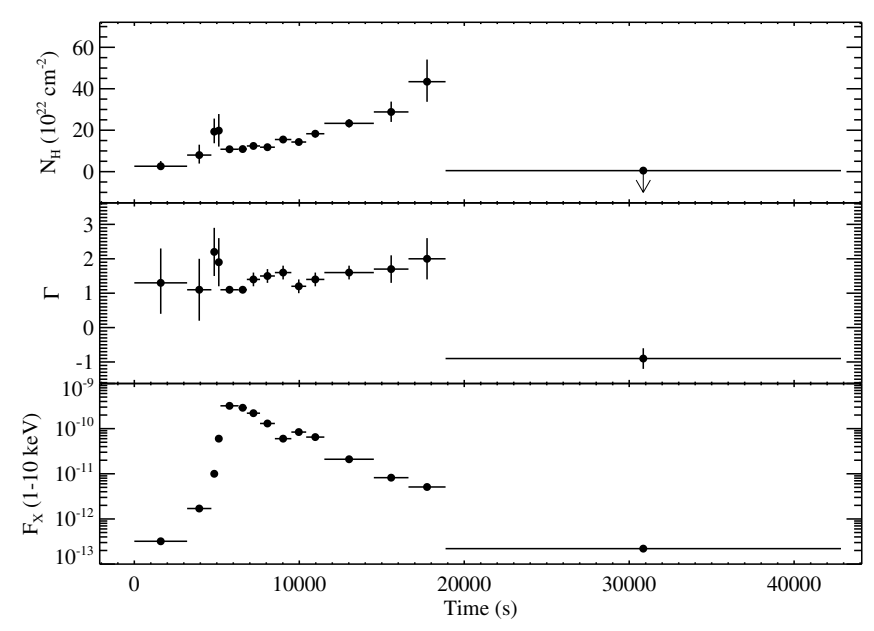

Fig. 7. Spectral parameters of IGR J18410-0535 measured during the different time intervals reported in Table 1 . All the errors are at $90 \%$ c.l. Arrows indicate $90 \%$ c.l. upper limits.

significance of the measured variation in the absorption column density, we report in Fig. 9 the $N_{\mathrm{H}}-\Gamma$ parameters confidence contours for some relevant spectra. In the upper panel of this figure, we show the parameter contours obtained by summing the spectra from intervals $\mathrm{C}$ and $\mathrm{D}$ and $\mathrm{A}$ and $\mathrm{B}$, which displayed very similar values of the power-law photon index and absorption column density before and during the rise of the flare, respectively (see Table 1). The contours correspond to $68 \%, 90 \%$, and $99 \%$ c.l. A very significant $(>3 \sigma)$ increase in the absorption column density at the onset of the flare is clearly seen. The lower panel of Fig. 9 shows the case of the $J$ and $N$ spectra: the increase in the absorption column density toward the end of the flare is also highly significant. During the apparent drop in count rate around $t=8500-9500 \mathrm{~s}$, no particular change in the spectral continuum is seen, but a significant iron line appears with a centroid energy of $\sim 6.4 \mathrm{keV}$ (see spectrum $I$ in Table 1 ; throughout this paper, we use narrow lines for the spectral fits and fixed their width to 0 in XSPEC).

The properties of the X-ray emission of the source changed sharply around $t \simeq 19000 \mathrm{~s}$ : at this time, the source underwent a further decrease in the X-ray flux, and simultaneously its spectrum flattened and a prominent iron emission line appeared around $6.4 \mathrm{keV}$. We show in Fig. 8 the detail of the evolution of the source spectrum with time during the latest $30 \mathrm{ks}$ of the $X M M-N e w t o n$ observation. A fit to the spectrum from the $\mathrm{O}$ interval with a simple absorbed power-law model gave unacceptable results $\left(\chi_{\text {red }}^{2} /\right.$ d.o.f. $\left.=2.1 / 15\right)$. Adding an iron line with an energy centroid of $E_{\text {line }}=6.36_{-0.05}^{+0.06} \mathrm{keV}$ significantly improved the fit $\left(\chi_{\text {red }}^{2} /\right.$ d.o.f. $\left.=1.0 / 13\right)$. The normalization and equivalent width of the line were $(2.7 \pm 1.0) \times 10^{-6}$ and $1.1 \mathrm{keV}$, respectively. From this spectrum, only an upper limit on $N_{\mathrm{H}}$ could be obtained (see Table 1); this was significantly lower than that expected in the direction of the source $\left(\sim 1.8 \times 10^{22} \mathrm{~cm}^{-2}\right.$, Dickey \& Lockman 1990). Even though this model gave a statistically acceptable fit to the spectrum $\mathrm{O}$, the very low value of the absorption column density and the unphysical negative power-law photon index suggest that other spectral models cannot be excluded. As we discuss in Sect. 5, a more physical interpretation of the X-ray emission of the source in this time interval is based 
IGRJ18410-0535 (epic-pn)

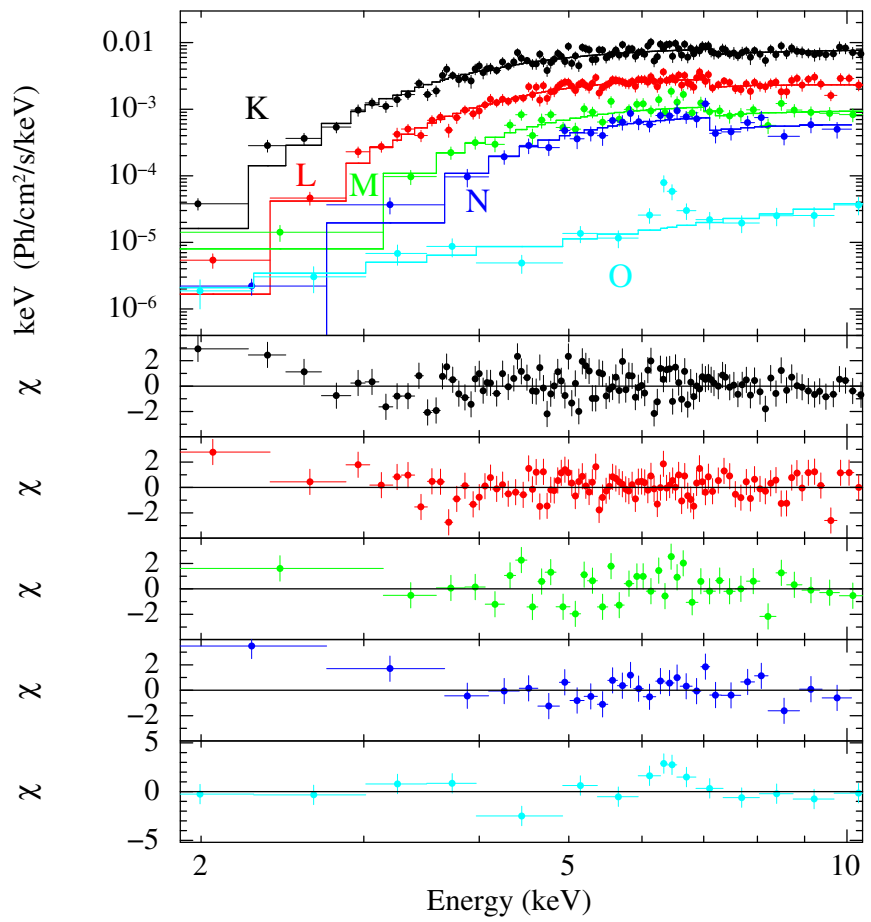

Fig. 8. XMM-Newton Epic-pn spectra of IGR J18410-0535 extracted during the latest part of the flare decay (see Table 1 and Fig. 4). All the spectra were fit with a simple absorbed power-law model (the residuals from these fits are shown for each of the five spectra).

on a model ${ }^{4}$ comprising two power-law components affected by two different absorption column densities plus the iron line, i.e. PHABS $1 *($ POW $1+$ GAUSS + PHABS $2 *$ POW 2$)$ in XSPEC. In the fit, we fixed phabs 1 to the Galactic value of the absorption column density and constrained the photon indices of the two powerlaws to be the same. The fit gave $\chi_{\text {red }}^{2}$ /d.o.f. $=0.8 / 12, \Gamma=0.9_{-0.5}^{+1.8}$ (in agreement with that measured during the other time intervals in Table 1), $E_{\text {line }}=6.36 \pm 0.06 \mathrm{keV}, E W=0.7 \mathrm{keV}$, and a value for the second absorption component of $\left(85_{-33}^{+48}\right) \times 10^{22} \mathrm{~cm}^{-2}$. In Fig. 10 we report the unfolded spectrum of interval $O$ together with the different spectral component used in this model.

In Fig. 11, we also show the contours plots of the iron line centroid energy versus its normalization for the three detections in the $E, I$, and $O$ spectra. We find marginal evidence for a decrease in the line centroid with time, from the peak of the flare (spectrum $E$ ) to the quiescent level (spectrum $O$ ). No other convincing evidence of the iron line could be found in the spectra extracted from different time intervals or from merging them in different combinations. The interpretation of all the above findings is discussed in Sect. 5.1.

We also performed a separate spectral analysis of the source $\mathrm{X}$-ray emission during the first $\sim 3 \mathrm{ks}$ and the last $\sim 17 \mathrm{ks}$ of the time interval $\mathrm{O}$ in order to investigate the cause of the drop in the source count-rate visible in Fig. 4 around $t=22820$. We indicate these two additional spectra with $\mathrm{O}_{1}$ (from $t=18870$ to $t=22820$ ) and $\mathrm{O}_{2}$ (from $t=22820$ to $t=42840$ ) in Table 1 .

\footnotetext{
${ }^{4}$ We also performed a fit using the reflection model PEXRAV in XSPEC, but the poor statistics of the $\mathrm{O}$ spectrum did not permit to derive satisfiable constraints on the model parameters. We thus do not discuss this model in further details.
}
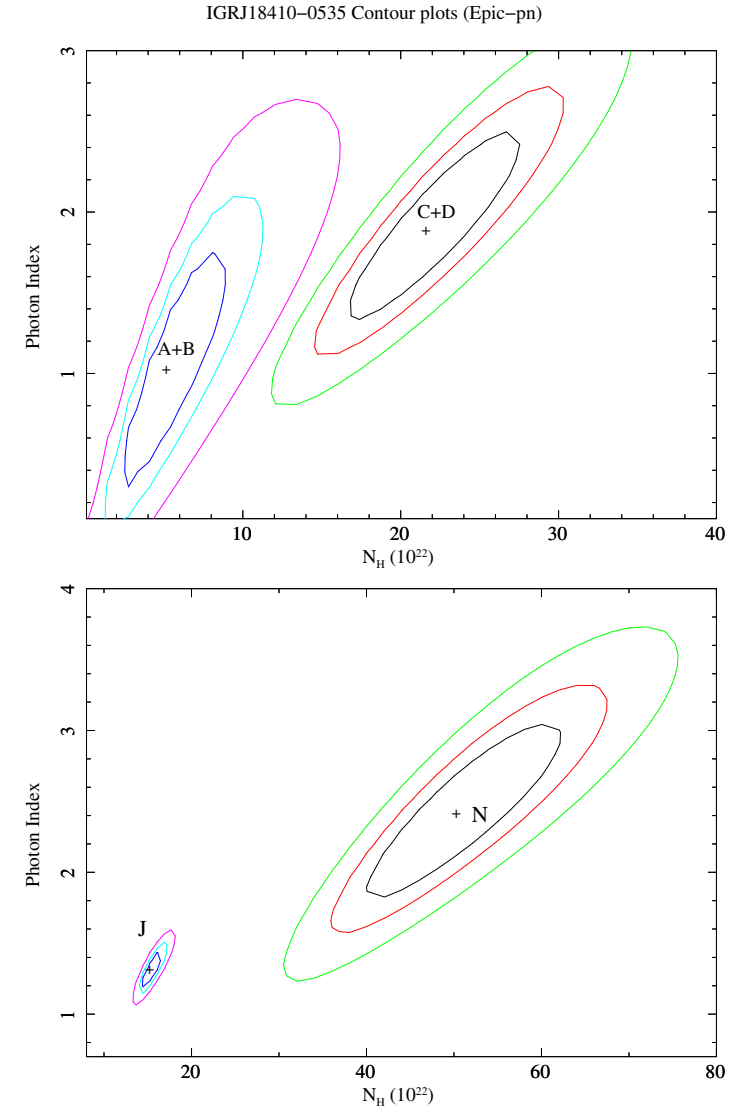

Fig. 9. Upper panel: contour plots for the spectral parameters measured from the time intervals $\mathrm{A}+\mathrm{B}$ and $\mathrm{C}+\mathrm{D}$ (see Table 1). The contours correspond to the $68 \%, 90 \%$, and $99 \%$ c.l. Lower panel: same as above but for the $J$ and $N$ spectra in Table 1 .

The $\mathrm{O}_{1}$ spectrum comprised 151 counts and was dominated by the presence of the iron line. A fit with a simple absorbed power-law did not provide an acceptable fit $\left(\chi_{\text {red }}^{2} /\right.$ d.o.f. $\left.=2.9 / 7\right)$, whereas adding a Gaussian line to the spectral model significantly improved the fit $\left(\chi_{\text {red }}^{2} /\right.$ d.o.f. $\left.=0.8 / 5\right)$. We measured a power-law photon index of $\Gamma=-1.8_{+0.6}^{-0.4}$, and a centroid energy for the line of $6.33_{-0.04}^{+0.06} \mathrm{keV}$. The normalization of the line was $(1.1 \pm 0.4) \times 10^{-5}$ and the estimated $E W 1.2 \mathrm{keV}$ (compatible with that found by using the entire time interval $\mathrm{O}$ ). Only an upper limit to the absorption column density of $N_{\mathrm{H}}<$ $8.0 \times 10^{22} \mathrm{~cm}^{-2}$ could be obtained.

Given the measured negative value of the parameter $\Gamma$, we also fit the spectrum $O_{1}$ by using the double power-law model discussed above and checked the consistency of the different model parameters with those determined before for the spectrum $O$. This gave $\Gamma=0.2_{-1.5}^{+1.0}$ and an absorption column density for the most extinguished power-law component of $82_{-42}^{+52} \times$ $10^{22} \mathrm{~cm}^{-2}$ (the absorption column density of the other component was fixed to the Galactic value). The centroid energy of the line and its $E W$ was found to agree with those reported before for the spectrum $O$. The estimated $1-10 \mathrm{keV} X$-ray flux was $9.7 \times 10^{-13} \mathrm{erg} \mathrm{cm}^{-2} \mathrm{~s}^{-1}$.

During the time interval $\mathrm{O}_{2}, X M M$-Newton recorded only 83 counts from the source. The corresponding spectrum could be closely fit with a simple absorbed power-law model (see Table 1). The quality of the fit did not change significantly (C-statistics 14.5/11) when the power-law photon index was constrained to be equal to that measured for the $O$ spectrum 
Table 1. Best-fit parameters of IGR J18410-0535 during different time intervals of the XMM-Newton observation (see Fig. 1 and the note below the table).

\begin{tabular}{lcccccccccc}
\hline \hline Interval & $\begin{array}{c}T \text { start } \\
(\mathrm{s})\end{array}$ & $\begin{array}{c}T \text { stop } \\
(\mathrm{s})\end{array}$ & $\begin{array}{c}\text { Exp. } \\
(\mathrm{ks})\end{array}$ & $\begin{array}{c}N_{\mathrm{H}} \\
\left(10^{22} \mathrm{~cm}^{-2}\right)\end{array}$ & $\Gamma$ & $\begin{array}{c}E_{\text {line }} \\
(\mathrm{keV})\end{array}$ & $\begin{array}{c}E W_{\text {line }} \\
(\mathrm{keV})\end{array}$ & $\begin{array}{c}F_{\text {obs }} \\
\left(\mathrm{erg} \mathrm{cm}^{-2} \mathrm{~s}^{-1}\right)\end{array}$ & $\begin{array}{c}F_{\text {unabs }} \\
\left(\mathrm{erg} \mathrm{cm}^{-2} \mathrm{~s}^{-1}\right)\end{array}$ & $\begin{array}{c}\chi_{\text {red }}^{2} / \mathrm{d} \text {.o.f. } \\
(\mathrm{C} \text {-statistics/d.o.f. })\end{array}$ \\
\hline $\mathrm{A}$ & 0 & 3200 & 2.7 & $2.6_{-1.7}^{+2.5}$ & $1.3_{-0.9}^{+1.0}$ & - & - & $3.2 \times 10^{-13}$ & $4 \times 10^{-13}$ & $(12.4 / 11)$ \\
$\mathrm{B}$ & 3200 & 4670 & 1.2 & $8.0_{-4.1}^{+5.0}$ & $1.1 \pm 0.9$ & - & - & $1.7 \times 10^{-12}$ & $2.8 \times 10^{-12}$ & $(14.4 / 18)$ \\
$\mathrm{C}$ & 4670 & 5020 & 0.35 & $19.3_{-5.6}^{+6.3}$ & $2.2 \pm 0.7$ & - & - & $1.0 \times 10^{-11}$ & $5.0 \times 10^{-11}$ & $(51.0 / 47)$ \\
$\mathrm{D}$ & 5020 & 5220 & 0.17 & $19.8_{-7.7}^{+8.0}$ & $1.9 \pm 0.7$ & - & - & $6.0 \times 10^{-11}$ & $2.4 \times 10^{-10}$ & $0.8 / 12$ \\
$\mathrm{E}$ & 5220 & 6320 & 0.96 & $10.8_{-0.6}^{+0.7}$ & $1.1 \pm 0.1$ & $6.56 \pm 0.05$ & 0.06 & $3.2 \times 10^{-10}$ & $6.0 \times 10^{-10}$ & $1.0 / 149$ \\
$\mathrm{~F}$ & 6320 & 6820 & 0.44 & $10.9_{-1.1}^{+1.2}$ & $1.1 \pm 0.1$ & - & - & $2.9 \times 10^{-10}$ & $5.3 \times 10^{-10}$ & $0.9 / 109$ \\
$\mathrm{G}$ & 6820 & 7620 & 0.7 & $12.4_{-1.2}^{+1.3}$ & $1.4 \pm 0.2$ & - & - & $2.2 \times 10^{-10}$ & $4.4 \times 10^{-10}$ & $1.1 / 108$ \\
$\mathrm{H}$ & 7620 & 8520 & 0.8 & $11.8_{-1.3}^{+1.5}$ & $1.5 \pm 0.2$ & - & - & $1.3 \times 10^{-10}$ & $2.9 \times 10^{-10}$ & $1.1 / 73$ \\
$\mathrm{I}$ & 8520 & 9520 & 0.9 & $15.5_{-1.5}^{+1.6}$ & $1.6 \pm 0.2$ & $6.32 \pm 0.05$ & 0.10 & $6.2 \times 10^{-11}$ & $1.8 \times 10^{-10}$ & $0.8 / 107$ \\
$\mathrm{~J}$ & 9520 & 10420 & 0.8 & $14.3_{-1.5}^{+1.4}$ & $1.2 \pm 0.2$ & - & - & $8.4 \times 10^{-11}$ & $1.9 \times 10^{-10}$ & $0.8 / 110$ \\
$\mathrm{~K}$ & 10420 & 11520 & 1.0 & $17.0 \pm 1.5$ & $1.3 \pm 0.2$ & - & - & $6.5 \times 10^{-11}$ & $1.6 \times 10^{-10}$ & $1.1 / 114$ \\
$\mathrm{~L}$ & 11520 & 14520 & 2.6 & $23.3 \pm 2.0$ & $1.6 \pm 0.2$ & - & - & $2.3 \times 10^{-11}$ & $8.5 \times 10^{-11}$ & $1.0 / 102$ \\
$\mathrm{M}$ & 14520 & 16620 & 1.8 & $28.0_{-4.8}^{+5.0}$ & $1.7 \pm 0.4$ & - & - & $8.2 \times 10^{-12}$ & $3.3 \times 10^{-11}$ & $1.2 / 58$ \\
$\mathrm{~N}$ & 16620 & 18870 & 1.8 & $43.4_{-9.7}^{+10.7}$ & $2.0 \pm 0.6$ & - & - & $5.1 \times 10^{-12}$ & $5.3 \times 10^{-11}$ & $1.4 / 36$ \\
$\mathrm{O}$ & 18870 & 42840 & 20.8 & $<0.5$ & $-0.9 \pm 0.3$ & $6.36 \pm 0.06$ & 1.1 & $2.2 \times 10^{-13}$ & $2.2 \times 10^{-13}$ & $1.0 / 13$ \\
$\mathrm{O}_{1}$ & 18870 & 22820 & 3.4 & $<8.0$ & $-1.8_{-0.4}^{+0.6}$ & $6.33 \pm 0.06$ & 1.2 & $9.7 \times 10^{-13}$ & $9.8 \times 10^{-13}$ & $0.8 / 5$ \\
$\mathrm{O}_{2}$ & 22820 & 42840 & 17.4 & $<1.4$ & $0.3_{-0.6}^{+0.5}$ & - & - & $8.8 \times 10^{-14}$ & $9.1 \times 10^{-14}$ & $(12.5 / 10)$ \\
\hline
\end{tabular}

Notes. The continuum spectral model is an absorbed power law. Here, $N_{\mathrm{H}}$ is the absorption column density, $\Gamma$ is the power-law photon index, $E_{\text {line }}$ is the energy of the centroid of the iron line, and $E W_{\text {line }}$ the corresponding equivalent width. $F_{\text {obs }}\left(F_{\text {unabs }}\right)$ is the absorbed (unabsorbed) flux in the 1-10 keV band. In the last column we report the value of the $\chi_{\text {red }}^{2} /$ d.o.f. or the corresponding value of the C-statistics/d.o.f, for each spectrum.

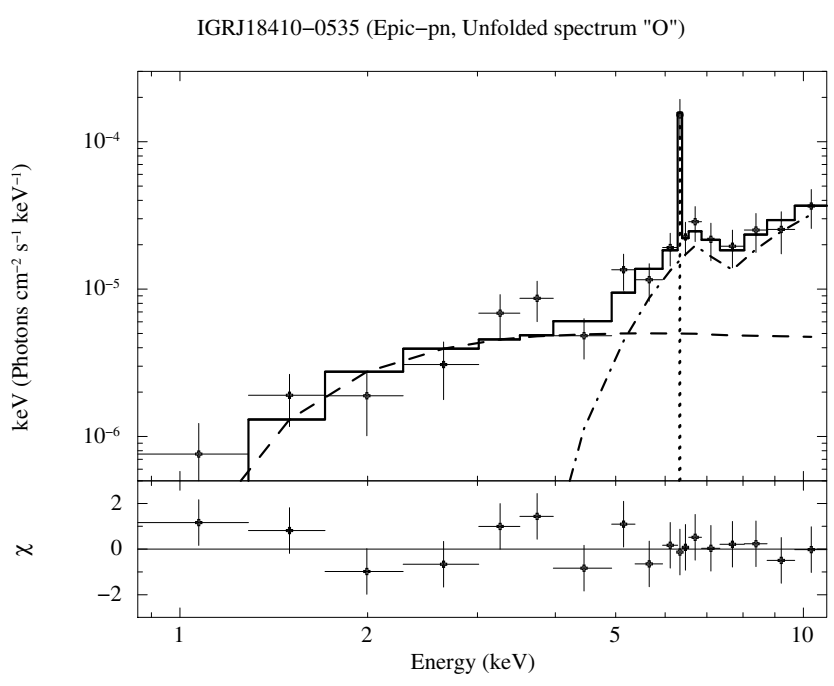

Fig. 10. Unfolded Epic-pn spectrum of IGR J18410-0535 during the interval $\mathrm{O}$ in Table 1 . The best-fit model in XsPEC here is phabs $1 *$ (pow $1+$ Gauss+phabs $2 *$ pow 2$)$. The value of phabs1 was fixed in the fit to $1.8 \times 10^{22} \mathrm{~cm}^{-2}$ and the photon index of the two power laws was forced to be the same (see Sect. 2.2 for details). The dashed line corresponds to the less absorbed power-law component in the fit, and the dot-dashed line to the more absorbed one. The dotted line indicates the iron line component at $\sim 6.4 \mathrm{keV}$. The bottom panel shows the residuals from the fit.

$(\Gamma=1.0)$; the corresponding value of the absorption column density was $N_{\mathrm{H}}=\left(1.6_{-1.0}^{+2.0}\right) \times 10^{22} \mathrm{~cm}^{-2}$, compatible with the expected Galactic value in the direction of the source. Given these results, we did not attempt to fit this spectrum with a double power-law model.
Adding to the spectral model an iron line with an energy fixed at $E_{\text {line }}=6.36 \mathrm{keV}$ provided an upper limit ( $90 \%$ c.l.) to its normalization and $E W$ of $1.8 \times 10^{-6}$ and $1.9 \mathrm{keV}$, respectively. While the upper limit to the $E W$ is consistent with that estimated for the $O$ spectrum, the normalization is slightly lower. However, it is still compatible with the value expected because of the decrease in the flux in the two spectra.

All these results suggest that the drop in the count-rate noticed around $t \sim 23000 \mathrm{~s}$ was accompanied by the occurrence of a further spectral change in the X-ray emission from the source. The spectrum $O_{1}$ could not be described well by assuming a simple absorbed power-law model and the addition of a second more absorbed component (plus the iron line) provided a more reasonable fit to the data. In contrast, the spectrum $\mathrm{O}_{2}$ could be well described by using a single relatively low absorbed power-law component. We show the two spectra $O_{1}$ and $O_{2}$ in Fig. 12, and discuss their interpretation further in Sect. 5.

We note finally that the relatively low quality statistics of spectra $\mathrm{A}$ and $\mathrm{O}_{2}$ and large errors affecting the values of their best-fit parameters prevent us from making a clear statement about any change in the spectral properties of the source before and after the occurrence of the flare. The XMM-Newton data revealed, however, that the flux of the source before the onset of the event (time interval A) was a factor $\sim 3.5$ higher that that estimated during the last part of the observation (time interval $\mathrm{O}_{2}$ ).

\subsection{Timing analysis}

IGR J18410-0535 was reported in previous studies to emit pulsations at a period of $4.7 \mathrm{~s}$ (Bamba et al. 2001; Romano et al. 2009; see also Sect. 1). We carried out an in-depth search for coherent modulations in data collected with both the Epic-pn 

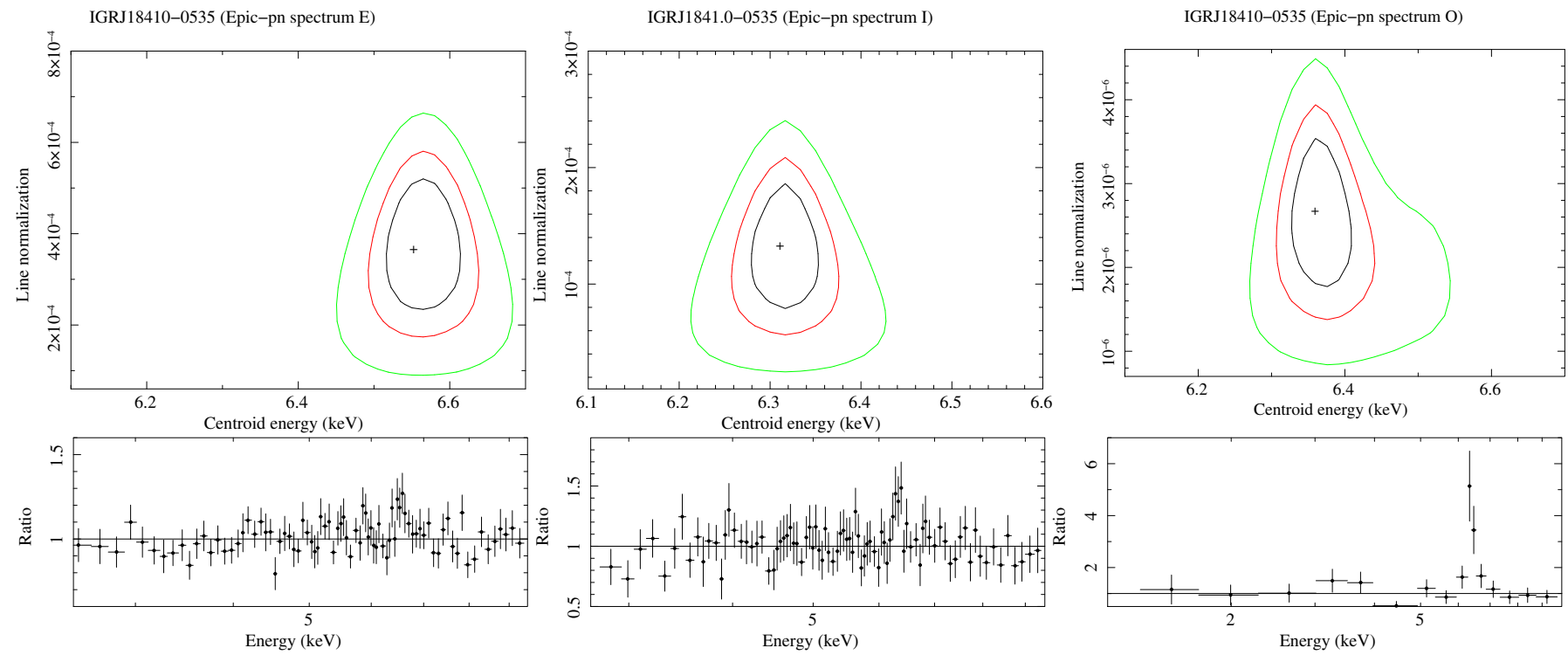

Fig. 11. Top panels: contour plots of the iron line centroid energy vs. normalization measured from the spectrum $E$ (left), I (middle), and $O$ (right). The contours correspond to the $68 \%, 90 \%$, and $99 \%$ c.l. There is a marginal indication of a change in the centroid energy of the line between the time interval $\mathrm{E}$ and the other two. Bottom panels: the ratio of the data to the model of the spectra extracted during the time interval $E$ (left), $I$ (middle), and $O$ (right). All these spectra were fit by using only an absorbed power-law model in order to highlight the presence of the iron line in the residuals from the fit.

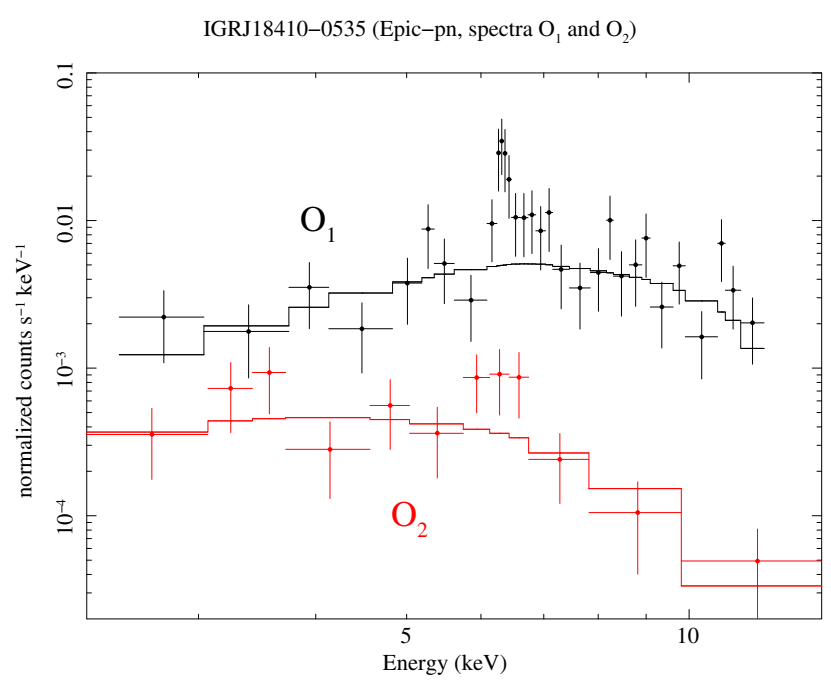

Fig. 12. The two spectra $\mathrm{O}_{1}$ and $\mathrm{O}_{2}$ extracted during the fist $\sim 3 \mathrm{ks}$ and the last $\sim 17 \mathrm{ks}$ of the time interval $\mathrm{O}$ in Table 1 . To facilitate the visual comparison, we used in this figure a grouping of 5 photons per bin for both spectra. We show the fit obtained with a simple power-law model to highlight the iron line at $\sim 6.4 \mathrm{keV}$.

and Epic-MOS cameras by extracting barycentered source and background event lists (we used the most accurately determined source position; see Sects. 1 and 2.3). We applied to the lists of barycentered photon arrival times in the $0.3-12 \mathrm{keV}$ energy range the power-spectrum search algorithm developed by Israel \& Stella (1996). This method is optimized to search for periodicities in "coloured" power spectrum components and derive upper limits if no signal is detected. Pulsations were searched for by using both the photon arrival times of the entire XMMNewton observation and in the different time intervals selected for the spectral analysis. We did not find any significant indication of pulsations. As an example, Fig. 13 shows the results obtained by applying the method of Israel \& Stella (1996) to the list of photon arrival times extracted from the entire XMM-Newton observation. In Table 2, we also report all the $3 \sigma$ upper limits on the pulsed fractions ${ }^{5}$ and pulsation frequencies we investigated. We show in this table the upper limit estimated by using $X M M-N e w t o n$ data accumulated during the entire observation $(\mathrm{A}+\mathrm{B}+\mathrm{C}+\mathrm{D}+\mathrm{E}+\mathrm{F}+\mathrm{G}+\mathrm{H}+\mathrm{I}+\mathrm{J}+\mathrm{K}+\mathrm{L}+\mathrm{M}+\mathrm{N}+\mathrm{O})$, the flare event (including rise and decay, $\mathrm{C}+\mathrm{D}+\mathrm{E}+\mathrm{F}+\mathrm{G}+\mathrm{H}$ ), the rise of the flare $(\mathrm{C}+\mathrm{D})$, the top of the flare $(\mathrm{E})$, the beginning of the flare decay $(\mathrm{F}+\mathrm{G}+\mathrm{H})$, the last part of the flare decay $(\mathrm{J}+\mathrm{K}+\mathrm{L})$, and the time interval in which the higher absorption was measured $(\mathrm{M}+\mathrm{N})$. This selection of intervals was carried out to investigate whether pulsations might have been present only during part of the event observed by XMM-Newton.

For all the time intervals reported in Table 2, we also performed additional searches of pulsations by separating the lists of photon time arrival times in the soft (0.3-4 keV) and hard (4$12 \mathrm{keV}$ ) energy bands and by combining strictly simultaneous EPIC-pn and MOS event lists (in this case, a common time resolution of 0.3-s was adopted). No significant improvement to the upper limits indicated in Table 2 could be obtained. Even though for a number of time intervals considered in Table 2 the derived $3 \sigma$ upper limit to the pulsed fraction was relatively high $(\sim 30$ $50 \%$ ), the entire exposure time of the XMM-Newton observation provided relatively tight constraints on the presence of pulsations from IGR J18410-0535 compared to those reported previously (see Sect. 1). Motivated by these findings, we reanalyzed all the published ASCA and Swift data for which detections of pulsations at $\sim 4.7 \mathrm{~s}$ were reported.

\section{ASCA data analysis}

IGR J18410-0535 was observed by ASCA for the first time on 1994 April 12 (MJD 49454.677-49454.701), during the survey of the Scutum arm region (hereafter Obs.1). A

${ }^{5}$ Here we define the pulsed fraction as the semi-amplitude of the sinusoid divided by the source average count rate, $\left(I_{\max }-I_{\min }\right) /\left(I_{\max }+I_{\min }\right)$. 
Table 2. Three $\sigma$ c.l. upper limits on the pulsed fraction of IGR J18410-0535 (see notes below the table).

\begin{tabular}{|c|c|c|c|}
\hline \multicolumn{4}{|l|}{ XMM-Newton $(0.3-12 \mathrm{keV})$} \\
\hline \multirow[t]{2}{*}{ Time interval } & \multicolumn{3}{|c|}{ Instrument (time resolution) } \\
\hline & PN (73.4 ms) & $\operatorname{MOS} 1(0.3 \mathrm{~s})$ & $\operatorname{MOS} 2(1.75 \mathrm{~ms})$ \\
\hline $\mathrm{A}+\mathrm{B}+\mathrm{C}+\mathrm{D}+\mathrm{E}+\mathrm{F}+\mathrm{G}+\mathrm{H}+\mathrm{I}+\mathrm{J}+\mathrm{K}+\mathrm{L}+\mathrm{M}+\mathrm{N}+\mathrm{O}$ & $6-10 \%(0.02-6.8 \mathrm{~Hz})$ & $10-20 \%(0.01-1.6 \mathrm{~Hz})$ & $15-30 \%(0.02-286 \mathrm{~Hz})$ \\
\hline $\mathrm{C}+\mathrm{D}+\mathrm{E}+\mathrm{F}+\mathrm{G}+\mathrm{H}$ & $10-30 \%(0.04-6.8 \mathrm{~Hz})$ & $10-30 \%(0.01-1.6 \mathrm{~Hz})$ & $10-30 \%(0.03-286 \mathrm{~Hz})$ \\
\hline $\mathrm{C}+\mathrm{D}$ & $30-50 \%(0.05-6.8 \mathrm{~Hz})$ & $50-70 \%(0.1-1.6 \mathrm{~Hz})$ & $60-80 \%(0.06-286 \mathrm{~Hz})$ \\
\hline $\mathrm{E}$ & $20-35 \%(0.05-6.8 \mathrm{~Hz})$ & $20-35 \%(0.03-1.6 \mathrm{~Hz})$ & $20-70 \%(0.1-286 \mathrm{~Hz})$ \\
\hline $\mathrm{F}+\mathrm{G}+\mathrm{H}$ & $15-20 \%(0.08-6.8 \mathrm{~Hz})$ & $20-30 \%(0.02-1.6 \mathrm{~Hz})$ & $20-30 \%(0.05-286 \mathrm{~Hz})$ \\
\hline $\mathrm{J}+\mathrm{K}+\mathrm{L}$ & $10-20 \%(0.02-6.8 \mathrm{~Hz})$ & $20-40 \%(0.01-1.6 \mathrm{~Hz})$ & $35-60 \%(0.02-286 \mathrm{~Hz})$ \\
\hline $\mathrm{M}+\mathrm{N}$ & $40-60 \%(0.02-6.8 \mathrm{~Hz})$ & $75-100 \%(0.02-1.6 \mathrm{~Hz})$ & $80-100 \%(0.02-286 \mathrm{~Hz})$ \\
\hline \multicolumn{4}{|l|}{$\operatorname{ASCA}(0.7-10 \mathrm{keV})$} \\
\hline & \multicolumn{3}{|l|}{ GIS $(0.5 \mathrm{~s})$} \\
\hline Obs. $1+$ Obs. 2 & \multicolumn{3}{|l|}{$30-45 \%(0.01-1 \mathrm{~Hz})$} \\
\hline
\end{tabular}

Notes. The three $\sigma$ upper limits on the pulsed fraction of IGR J18410-0535 are determined for the three Epic cameras in the different time intervals reported in Table 1. The results of a similar analysis carried out on the ASCA data are also reported (see Sect. 3.1 for the discussion of the ASCA data analysis).

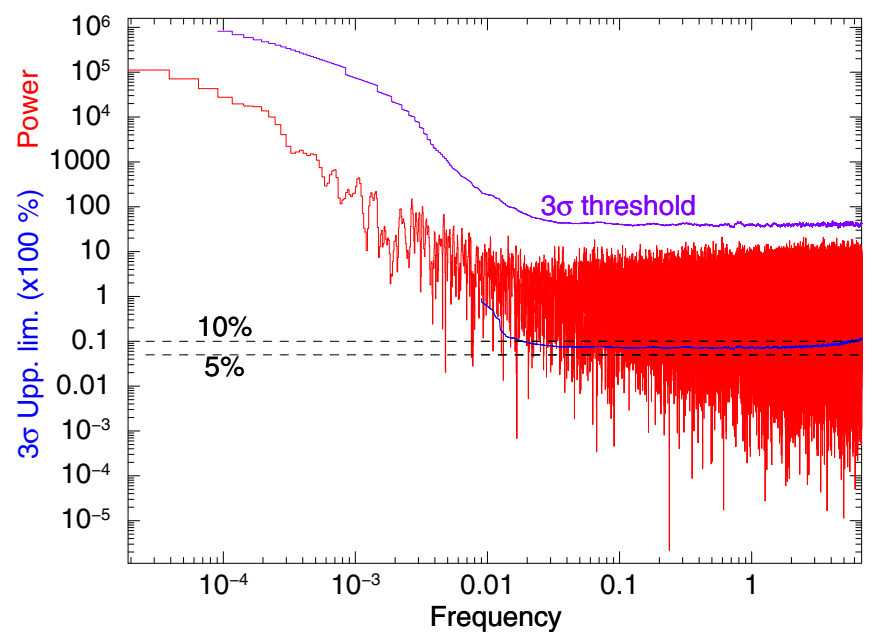

Fig. 13. Power spectrum produced using data from the entire $X M M$ Newton observations $(0.3-12 \mathrm{keV})$. The upper continuous line represents the power threshold for detection of periodicity at a $3 \sigma$ c.l., according to the method described by Israel \& Stella (1996). We also show in the bottom part of the plot a second continuous curve that represents the upper limit calculated with the same method, on the presence of pulsations from IGR J18410-0535 as a function of the frequency. The two dashed lines represent the 5\% and 10\% upper limit levels on that curve. The most stringent upper limit we could provide with this method is $6-10 \%$ for frequencies in the range $0.02-6.8 \mathrm{~Hz}$.

follow-up observation was carried out on 1999 October 3-4 (MJD 51 454.252-51455.463, hereafter Obs. 2). On both occasions, IGR J18410-0535 was outside the field of view (FOV) of the Solid-state Imaging Spectrometers (SIS, Burke et al. 1993), but included in that of Gas Imaging Spectrometers (GIS, Ohashi et al. 1996). The GISs were operated in the nominal PH mode with a time resolution of $62.5 \mathrm{~ms}$ for data taken in high bit-rate and $0.5 \mathrm{~s}$ for those taken in medium bit-rate. For our analysis, we used only GIS data obtained when the satellite was either outside the South Atlantic Anomaly and low cut-off rigidity regions $(>6 \mathrm{GV})$, or when the target elevation angle was $>5^{\circ}$. Particle events were removed based on rise-time discrimination with the GISCLEAN task (Ohashi et al. 1996). After this screening, the total available exposure times of Obs. 1 and 2 were $2 \mathrm{ks}$ and $36 \mathrm{ks}$, respectively. To improve the quality of the statistics, we combined data from the GIS-2 and GIS-3 detectors. ASCA events were extracted from a circular region of 3 arcmin radius centered on the best known source position (Halpern et al. 2004). The background was extracted from an annular region around the source position with inner radius of $4.3 \mathrm{arcmin}$ and outer radius of 6.8 arcmin. This annular region is free from field point sources and is unaffected by the extended diffuse emission from the nearby supernova remnant G26.6-0.1 (Bamba et al. 2003). The inner radius of the background extraction radius was chosen so as to avoid contamination due to the tail of the ASCA PSF. Photon arrival times were all converted to the Solar System barycenter (SSB) with the task TIMECONV using the Chandra position reported by Halpern et al. (2004). The $\log$ of the ASCA observations of IGR J18410-0535 is given in Table 3.

\subsection{Timing analysis}

In Fig. 14, we show the background-subtracted light curve (top panel) of IGR J18410-0535 in the $0.7-10 \mathrm{keV}$ energy band. According to the results reported by Bamba et al. (2001), we note that the source was characterized by a variable intensity, and that a $5 \mathrm{hr}$-long X-ray flare is visible at the end of Obs. 2 (starting from MJD 51455.3). During this flare, the intensity of the source increased by a factor of $\sim 5$. After $\sim 14 \mathrm{ks}$, IGR J18410-0535 returned to an X-ray emission level comparable to that observed before the flare. The bottom panel of Fig. 14 shows the evolution of the hardness ratio computed by using the lightcurves extracted in the $0.7-2 \mathrm{keV}$ and 2-10 keV energy bands. In Obs. 2, the HR showed a remarkable increase (from $\sim 0.5$ to $\sim 2$ ) with the source intensity.

During the flare in Obs. 2, Bamba et al. (2001) reported on the detection of coherent pulsations by carrying out a fast Fourier transform (FFT) of the barycentered photons-event arrival times selected in the 1.9-4.9 keV energy range (energy channels 161415) and extracted from a $3^{\prime}$ circular region centered on the best known source position. An excess in the power spectrum 
Table 3. ASCA observations log of IGR J18410-0535.

\begin{tabular}{lcccc}
\hline \hline Instrument & Observation & Start time & Time elapsed & Exposure \\
\hline ASCA (1) & 61009100 & $1994-12-04 T 15: 48: 13$ & 4312.0 & 2112.0 \\
ASCA (2) & 57026000 & $1999-10-03 T 05: 47: 32$ & 65484.0 & 37373.6 \\
\hline
\end{tabular}

Table 4. Swift observations log of IGR J18410-0535.

\begin{tabular}{lcccc}
\hline \hline Obs. ID & Instr. & $\begin{array}{c}\text { Start time } \\
\text { (UTC) }\end{array}$ & $\begin{array}{c}\text { Stop time } \\
\text { (UTC) }\end{array}$ & $\begin{array}{c}\text { Exp. } \\
(\mathrm{ks})\end{array}$ \\
\hline 00030988001 & XRT/PC & $2007-10-2600: 08$ & $2007-10-2606: 45$ & 1.4 \\
00030988004 & XRT/PC & $2007-11-0509: 08$ & $2007-11-0509: 28$ & 1.2 \\
00030988005 & XRT/PC & $2007-11-0916: 11$ & $2007-11-0916: 32$ & 1.3 \\
\hline
\end{tabular}

Notes. Exp. indicates the effective exposure time of the observation.

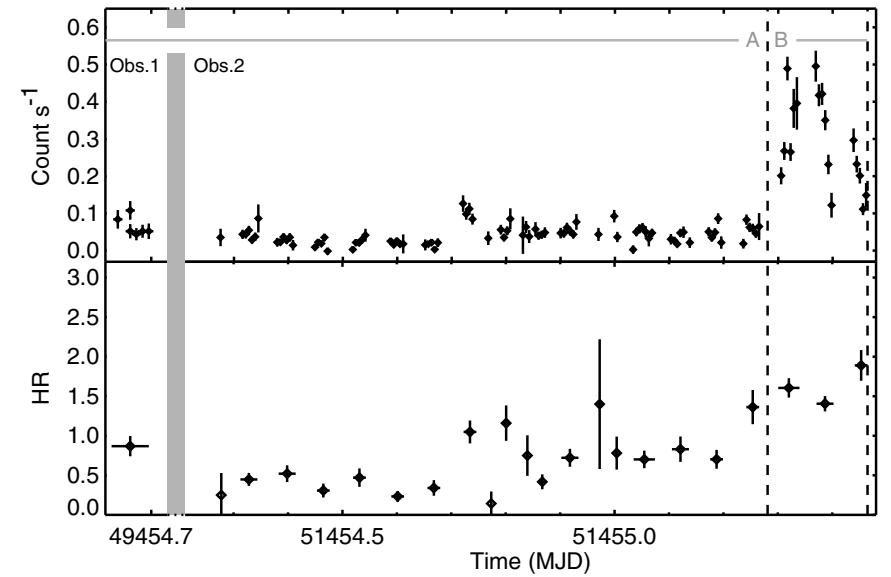

Fig. 14. Top panel: ASCA light curve of IGR J18410-0535 in the 0.7$10 \mathrm{keV}$ energy band. Bottom panel: hardness ratio of the 4-10 and 1$4 \mathrm{keV}$ GIS light curves. The vertical dashed lines mark the flare episode starting at MJD 51 455.3. The hashed region marks the separation between Obs. 1 and Obs. 2. A and B denote the two data set selected for the timing analysis.

$(0.02-1 \mathrm{~Hz})$ of $\sim 40$ was visible at $\sim 0.211 \mathrm{~Hz}$ (their most accurately determined spin period was $P=4.7394 \pm 0.0008 \mathrm{~s}$, see also Sect. 1). Taking into account the total number of independent frequency trials, this excess corresponds to 4.4 (Gaussian) standard deviations.

We revisited the data from Obs. 2 performing a power spectrum analysis. The source events were collected during the flare with a time bin of $0.5 \mathrm{~s}$ (the time resolution of medium bitrate ASCA data) and extracted from a 3' radius circular region around the source. We investigated frequencies in the range $0.02-1 \mathrm{~Hz}$ with a resolution of $3.7 \times 10^{-5} \mathrm{~Hz}$, first by using the entire energy range of the GIS (i.e. $0.7-10.0 \mathrm{keV}$ ) and then only the events in the $1.9-4.9 \mathrm{keV}$ and $4.9-10.0 \mathrm{keV}$ energy intervals.

In the first and the latter cases, the power spectrum did not reveal any significant deviations from a statistically flat distribution. The corresponding estimated upper limit on the pulsed fraction of the source at different frequencies is reported in Table 2 (we used the method described in Sect. 2.3 in order to facilitate the comparison with the XMM-Newton results). The power spectrum realized with the events in the energy interval 1.9$4.9 \mathrm{keV}$ showed a peak at $\sim 0.211 \mathrm{~Hz}$ with a power of $\sim 33$ (see
Fig. 15). If all the inspected independent frequencies and the ad-hoc selection in energy are taken into account to estimate the total effective number of trials, this peak corresponds to a detection significance of $\$ 3$ (Gaussian) standard deviations.

A signcance of $\sim 4.4$ (Gaussian) standard deviations for pulsations at $\sim 4.7 \mathrm{~s}$ could be obtained only by applying the FFT to the $1.9-4.9 \mathrm{keV}$ flare events and using a time bin of $1 \mathrm{~s}$. Different choices of the time binning would led to a result comparable to that obtained with our Fourier analysis (detection signcance 3 sigma).

Since pulsations in the ASCA data seems to be detected with a suciently high statistical significance only when a specic energy range is selected and only performing the analysis with the FFT technique and an ad-hoc time bin of $1 \mathrm{~s}$, we conclude that this detection could be due to a statistical fluctuation.

\section{Swift data analysis}

Pulsations in the X-ray emission of IGR J18410-0535 were also reported by Sidoli et al. (2008). These authors used the three Swift/XRT observations ID. 00030988001, 00030988004, and 00030988005 (see Table 4). All data were collected in photon counting mode (PC, time resolution $2.5 \mathrm{~s}$ ), and summed up to extract a single source photon event list and increase the statistics. By using a folding technique on this event list, Sidoli et al. (2008) found a peak in the periodogram at $4.70088 \pm 0.0004 \mathrm{~s}$ with a reduced $\chi^{2}$ of 6.3 ( 9 degree of freedom).

\subsection{Timing analysis}

We reanalyzed the Swift XRT data of the above Swift-XRT observations to verify the detection of pulsations. We used the same Swift data analysis described in Sidoli et al. (2008). XRT data were processed with standard procedures (xrtpipeline v. 0.11.6). Filtering and screening criteria were applied by using FTOOLS and only event grades $0-12$ were considered. We extracted a source event list (194 events) by using a circular region of 29 arcsec centred on the best known position of IGR J18410-0535 (Halpern et al. 2004) and a background event list (1106 events) by using an annular region centered on the same position and with inner radius of 245 arcsec and outer radius of 630 arcsec. The radii of the background extraction region were chosen in order to avoid any contamination from the tail of the $\mathrm{PSF}^{6}$. The

6 See also http://heasarc.gsfc.nasa.gov/docs/swift/ analysis/xrt_swguide_v1_2.pdf. 


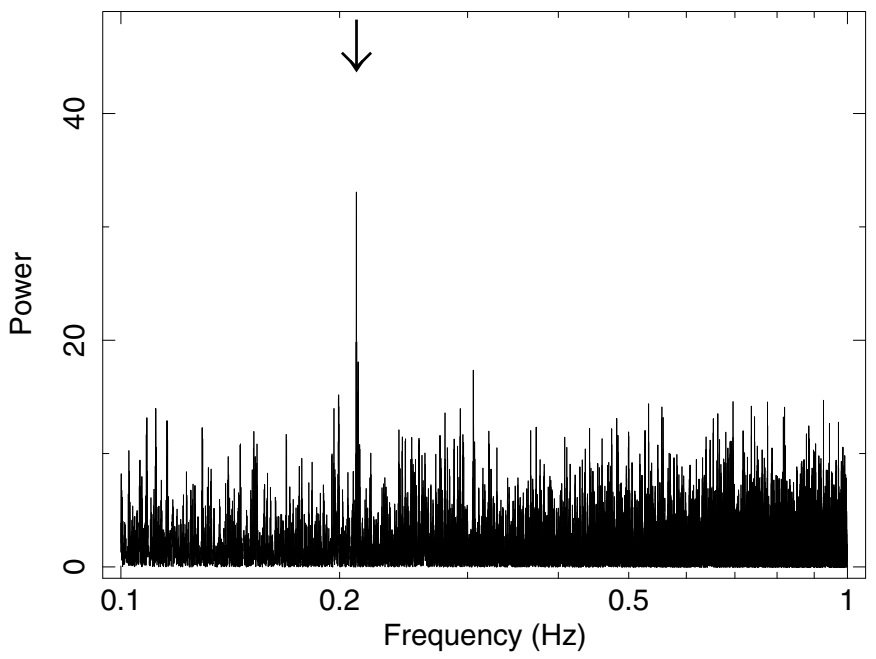

Fig. 15. ASCA power spectrum of IGR J18410-0535 obtained by using events in the 1.9-4.9 keV energy range and a time resolution of $0.5 \mathrm{~s}$. The arrow marks the apparent detection of pulsations at $0.211 \mathrm{~Hz}$. As we discuss in the text, this excess in the power-spectrum is not statistically significant if the correct number of trials is taken into account (see Sect. 3.1).

arrival times of the source and background events were all converted to the SSB with the task BARYCORR. We applied the folding technique adopted by Sidoli et al. (2008) to the source and background event lists, to search for a period of $\sim 4.7 \mathrm{~s}$. In the periodogram obtained from the analysis of source events (Fig. 16, upper panel $)$ the peak at $4.70088\left(\chi_{\text {red }}^{2} /\right.$ d.o.f. $\left.=6.5 / 9\right)$ is visible. However, we noted that the same peak is also present in the periodogram obtained from the background event list (Fig. 16, lower panel). In this case, we obtained $\chi_{\text {red }}^{2}=23$. The difference in the value of the $\chi_{\text {red }}^{2}$ (a factor $\sim 4-5$ ) is consistent with the different quality of the statistics content of the two data samples. We thus argue that the detection of pulsations reported by Sidoli et al. (2008) was merely due to an instrumental effect present in the photon arrival times of the Swift/XRT data. Investigations of similar effects in other Swift/XRT data-sets are currently going on and will be reported in the relevant on-line pages ${ }^{7}$.

\section{Discussion}

During the $45 \mathrm{ks}$-long XMM-Newton observation presented here, IGR J18410-0535 underwent a bright X-ray flare lasting about $15 \mathrm{ks}$. The rise of the flare occurred $5 \mathrm{ks}$ after the beginning of the observation, thus we were able to follow in detail the whole event to the return to quiescence. A time-resolved spectral analysis of the observation revealed that the source X-ray emission could be described well in the different selected time intervals by using an absorbed power-law model. The photon index of the power law remained virtually constant at least for the first $20 \mathrm{ks}$ of the observation, but in this period the absorption column density underwent dramatic changes. We measured a significant rise in the $N_{\mathrm{H}}$ from $\sim 3$ to $20 \times 10^{22} \mathrm{~cm}^{-2}$ across the transition from quiescence to the peak of the flare, followed by a sudden decrease to a value of $10 \times 10^{22} \mathrm{~cm}^{-2}$ which then remained stable for the following $4 \mathrm{ks}$. In the subsequent $11 \mathrm{ks}$, the $N_{\mathrm{H}}$ progressively increased to a value of $\sim 50 \times 10^{22} \mathrm{~cm}^{-2}$, and the spectral properties of the source changed dramatically

\footnotetext{
7 http://www.swift.ac.uk/xrtdigest.shtml
}
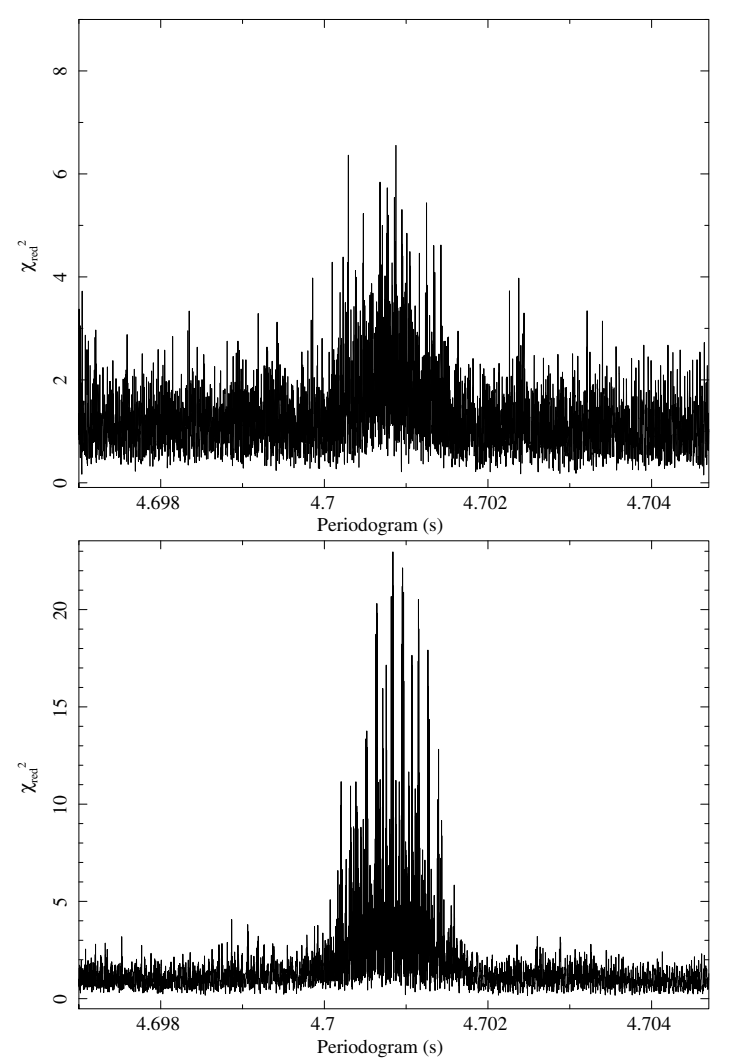

Fig. 16. Periodogram realized with the Swift XRT data by using the source (upper panel) and background (lower panel) event lists extracted by summing up data in the three observations ID. 00030988001 , 00030988004 , and 00030988005 .

during the last $23 \mathrm{ks}$ of observation. In this time interval, we measured an abrupt flattening of the source power-law photon index and revealed the appearance of a prominent emission line at $\sim 6.4 \mathrm{keV}$. Similar behavior is usually observed during the occurrence of X-ray eclipses in some of the sgHMXBs and in only one SFXT (IGR J16479-4514, see e.g., Bozzo et al. 2008b, and references therein). When the NSs in one of these systems is obscured by its supergiant companion, the X-ray emission produced by the accretion process close to the surface of the compact object is progressively absorbed in the photosphere of the supergiant star. Only the X-rays reflected by matter in the stellar wind remain visible to the observer and a fluorescence emission line at $\sim 6.4 \mathrm{keV}$ becomes very prominent.

In the present case, that IGR J18410-0535 underwent a bright X-ray flare after which the source turned off sharply because of an X-ray eclipse appears to be unlikely. Below we discuss an alternative scenario, in which the entire event observed by XMM-Newton is interpreted as the accretion of a massive clump onto the compact object hosted in IGR J18410-0535.

\subsection{Ingestion of a massive clump}

The idea that dense "clumps" of matter might be generated in the wind of supergiant stars as a consequence of different magnetohydrodynamic instabilities has found some support in the results of several numerical simulations (Runacres \& Owocki 2002; Oskinova et al. 2007, and references therein) and in observations of isolated OB stars (see e.g., Eversberg et al. 1998; Martins 2011). Even though the physical parameters of these clumps (i.e., mass, radius and density) are still largely unknown 

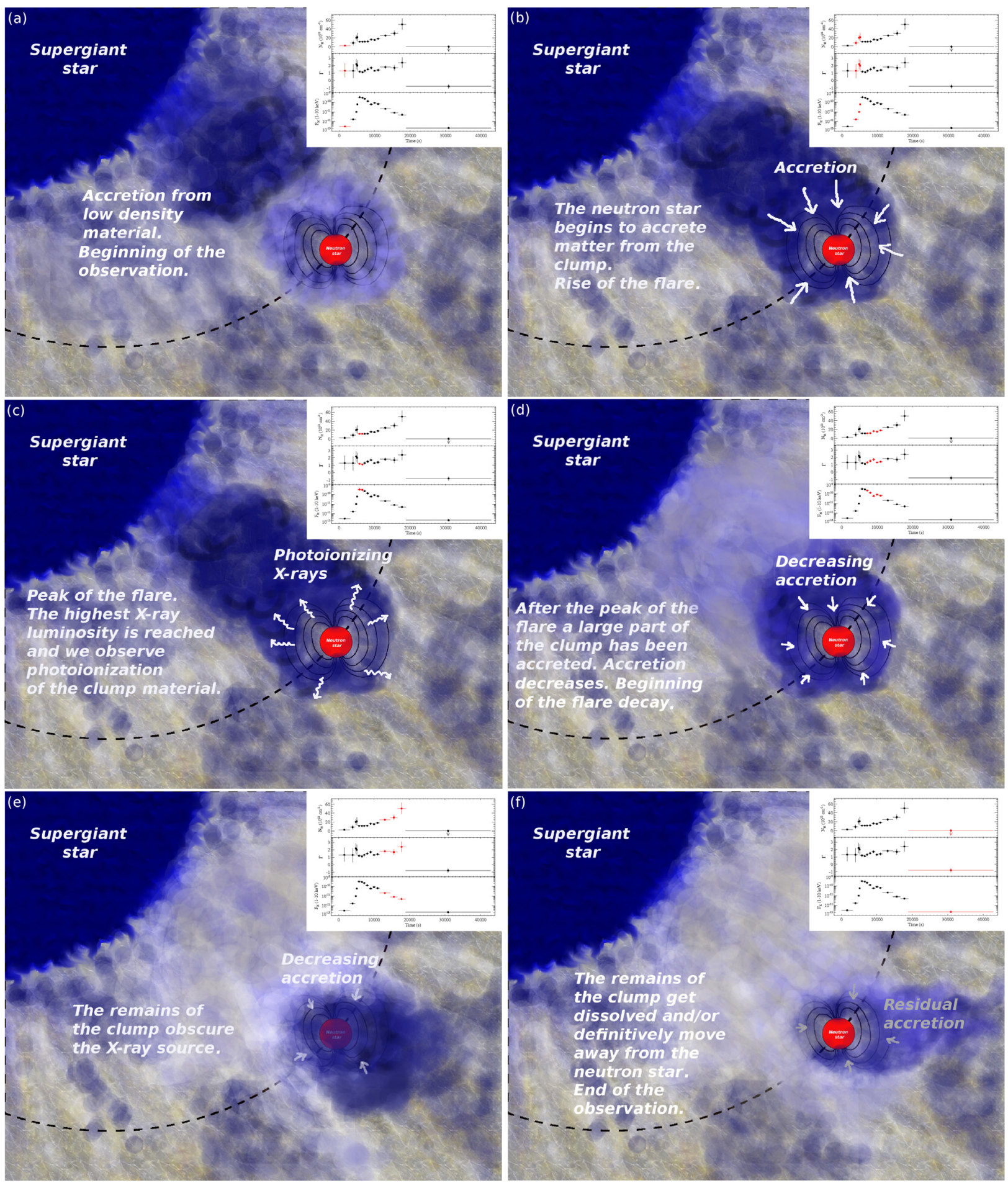

Fig. 17. An artist's view of the "ingestion of a clump" described in Sect. 5.1. Different elements in the figure are not represented to scale. The figures illustrate qualitatively the main phases of the event in chronological order from the top left to the bottom right. In each figure, we plot in the top right corner the XMM-Newton lightcurve of the event already shown in Fig. 7 and highlight with a red color the data points in the plot that correspond to the physical scenario described in the picture. From top left to bottom right: a) at the beginning of the XMM-Newton observation the NS is accreting from a low density material, such that the observed X-ray luminosity is relatively low $\left(\sim 4 \times 10^{32} \mathrm{erg} \mathrm{s}^{-1}\right)$; b) the NS encounters the clump and XMM-Newton observes a rapid rise in the X-ray flux from the source; c) the higher X-ray luminosity $\left(\sim 4 \times 10^{35} \mathrm{erg} \mathrm{s}^{-1}\right)$ causes the photoionization of the surrounding material and a decrease in $N_{\mathrm{H}} ; \mathbf{d}$ ) accretion decreases and the source undergoes a rapid decay in the X-ray flux; e) the remains of the clump move in front of the NS along the observer line of sight and obscure the X-ray source; f) the remains of the clump move away and the source returns to its quiescent state $\left(\sim 10^{32} \mathrm{erg} \mathrm{s}^{-1}\right.$, some residual accretion might still take place).

because of poorly estimated theoretical and observational parameters, a clumpy wind scenario is the founding hypothesis of most models developed so far to interpret the X-ray variability of the SFXTs (in't Zand 2005; Walter \& Zurita Heras 2007;
Bozzo et al. 2008a, and references therein). All these models predict that fast X-ray flares can be produced by the sporadic capture and accretion of one of these clumps onto the NSs hosted in the SFXTs. The role of these dense clumps distributed all 
around the NS orbit is two-fold. Clumps simply passing in front of the X-ray source cause dimming or even obscuration, and display the signatures of photoelectric absorption, at least during the ingress and egress stages. In addition to these phenomena, clumps that lead to increased accretion also give rise to large variations in the X-ray luminosity. To reach the required X-ray luminosity level, different densities of the clumps might be required, depending also on the strength of the NS magnetic field and the value of its spin period. Both the rotation of the NS and the extent of its magnetosphere can act as gates that halt most of the accretion during quiescence and release it during the outburst, thus significantly altering the total dynamic range of the Xray luminosity (Grebenev \& Sunyaev 2007; Bozzo et al. 2008a).

In the case of IGR J18410-0535, the entire flaring episode observed by XMM-Newton might be due to the accretion of a single massive clump.

$-t=0-3200 \mathrm{~s}$ (time interval A): Pre-flare quiescence. At the beginning of the XMM-Newton observation, the source was caught at a relatively low X-ray flux $\left(3.2 \times 10^{-13} \mathrm{erg} \mathrm{cm}^{-2} \mathrm{~s}^{-1}\right)$. For a source distance of $3 \mathrm{kpc}$, this would translate into an X-ray luminosity of $3.5 \times 10^{32} \mathrm{erg} / \mathrm{cm}^{2} \mathrm{~s}^{-1}$, i.e. a value typical of SFXTs in quiescence. The spectral parameters, $N_{\mathrm{H}}, \Gamma$, that we measured in this time interval (spectrum A in Table 1) are compatible with those inferred for the "very low state" reported by Romano et al. (2009). The relatively low flux and absorption column density measured in this state with respect to the other average X-ray emission states ("medium" and "high") suggests that the NS at the beginning of the XMM-Newton observation might have been located in a tenuous region of the stellar wind such that accretion was taking place only at a very low level or that it was inhibited by magnetic and/or centrifugal barriers.

- $t=3200-5220 \mathrm{~s}$ (time intervals B, C, and D): Rise of the flare. Around $t=3200 \mathrm{~s}$, the XMM-Newton lightcurve showed a remarkable rapid increase in the count-rate from IGR J18410-0535. This is firstly noticeable in the hard energy band (4-12 keV, see Fig. 2), thus indicating a high level of absorption. During the rise of the flare, we measured a significant increase in $N_{\mathrm{H}}$, reaching a value of $\sim 2 \times 10^{23} \mathrm{~cm}^{-2}$ at the top of the flare $(t \simeq 5200 \mathrm{~s})$. At this stage, the source $\mathrm{X}$-ray flux increased to $3.3 \times 10^{-10} \mathrm{erg} \mathrm{cm}^{-2} \mathrm{~s}^{-1}$, corresponding to an X-ray luminosity of $3.6 \times 10^{35} \mathrm{erg} \mathrm{s}^{-1}$. A similar simultaneous increase in the X-ray luminosity and absorption column density can be well understood by assuming that a massive clump approached the NS magnetosphere and filled the immediate surroundings with high density material. The increase in the local density can easily lead to a significant increase in the $N_{\mathrm{H}}$ along our line of sight and eventually compress the NS magnetosphere to open the magnetic and/or centrifugal barrier and permit direct accretion onto the compact object. In this interpretation, we can use the observational results in Table 1 to estimate the physical properties of the clump.

In wind accretion theory, the typical time scale on which matter is accreted and reaches the surface of the NS from the magnetospheric boundary is of the order of the local free-fall time, i.e. hundreds of seconds (see e.g., Bozzo et al. 2008a). The duration of the flare observed from IGR J18410-0535 is $\sim 15 \mathrm{ks}$, thus this time is likely to be linked to the radial extent of the clump. Therefore, we can estimate

$R_{\mathrm{cl}} \simeq 1 / 2 v_{\mathrm{w}} \Delta t_{\text {flare }}=8 \times 10^{11} v_{\mathrm{w} 8} \mathrm{~cm}$, where $R_{\mathrm{cl}}$ is the radius of the clump and $v_{\mathrm{w} 8}$ is the relative velocity between the clump and the NS in units of $10^{8} \mathrm{~cm} \mathrm{~s}^{-1}$ (we neglect here the orbital velocity of the NS and consider a spherical clump moving with the same velocity as the surrounding stellar material; Lépine \& Moffat 2008). To a first order approximation, only the material that falls inside the accretion radius, $R_{\mathrm{acc}}$, of the NS is accreted, and thus

$M_{\mathrm{cl}}=\left(R_{\mathrm{cl}} / R_{\mathrm{acc}}\right)^{2} M_{\mathrm{acc}}$,

where $R_{\mathrm{acc}}=2 G M_{\mathrm{NS}} / v_{\mathrm{w}}^{2}, M_{\mathrm{NS}}=1.4 M_{\odot}$ is the mass of the NS, and $M_{\text {acc }}$ is the total mass accreted during the flare. The latter can be estimated by integrating the X-ray flux measured during the flare as a function of time in Fig. 7 and using the relations $F_{\text {unabs }}=L_{\mathrm{X}} /\left(4 \pi d^{2}\right)$ and $L_{\mathrm{X}}=G M_{\mathrm{NS}} \dot{M}_{\mathrm{acc}} / R_{\mathrm{NS}}$ (where $d$ is the source distance, $R_{\mathrm{NS}}=10^{6} \mathrm{~cm}$ is the NS radius and $\dot{M}_{\text {acc }}$ is the mass accretion rate). We found $M_{\text {acc }}=$ $1.5 \times 10^{19} d_{3 \mathrm{kpc}} \mathrm{g}$ (where $d_{3 \mathrm{kpc}}$ is the source distance in units of $3 \mathrm{kpc}$ ) and thus

$M_{\mathrm{cl}}=1.4 \times 10^{22} v_{\mathrm{w} 8}^{6} d_{3 \mathrm{kpc}}^{2} \mathrm{~g}$,

which clearly has a strong dependence on the wind velocity. Another equation that can be used as a cross-check of these results is that relating the mass and radius of the clump to the expected absorption column density caused by its presence in the vicinity of the NS, i.e.

$N_{\mathrm{H}} \simeq M_{\mathrm{cl}} /\left(R_{\mathrm{cl}}^{2} m_{\mathrm{p}}\right)=1.3 \times 10^{22} v_{\mathrm{w} 8}^{4} d_{3 \mathrm{kpc}}^{2} \mathrm{~cm}^{-2}$.

As the $N_{\mathrm{H}}$ measured by the spectral analysis at the top of the flare is $\sim 2 \times 10^{23} \mathrm{~cm}^{-2}$, Eq. (4) implies a wind velocity slightly lower than $10^{8} \mathrm{~cm} \mathrm{~s}^{-1}$. We note that the estimated values of $M_{\mathrm{cl}}$ and $R_{\mathrm{cl}}$ are in qualitative agreement with those expected according to the clumpy wind model developed by Ducci et al. (2009).

- $t=5220-6320 \mathrm{~s}$ (time interval E): Top of the flare. The results of the spectral analysis obtained during this time interval can also be clearly explained within the scenario depicted above. The time-resolved spectral analysis carried out in Sect. 2.2 showed that the initial rise in the $N_{\mathrm{H}}$, interpreted above as being due to a massive clump approaching the NS, was followed by a sudden decrease in the absorption column density (by a factor of $\sim 2$ ) when the system reached the highest luminosity. According to the calculation of Krolik \& Kallman (1984), a similar drop in $N_{\mathrm{H}}$ is to be expected because of the heating and photoionization of the clump material by the higher X-ray flux. In particular, a major change in the opacity of the material with respect to the X-ray photons is expected when the so-called ionization parameter $\Xi \simeq 10$. The latter is defined as

$\Xi=\frac{L_{\mathrm{X}}}{4 \pi r^{2} N k T} \simeq 8 v_{\mathrm{w} 8}^{-5} d_{3 \mathrm{kpc}}^{-2} L_{35} T_{5}^{-1}$,

where $L_{35}=L_{\mathrm{X}} /\left(10^{35} \mathrm{erg} \mathrm{s}^{-1}\right)$ is the luminosity coming from the $\mathrm{X}$-ray source, $r \simeq R_{\mathrm{cl}}$ is the distance of the material from the source, and $T \simeq 10^{5} \mathrm{~K}$ is the temperature of the clump (Ducci et al. 2009). We therefore expect $\Xi$ to be close to the critical value for a luminosity similar to that reached by IGR J18410-0535 at the peak of the flare. During this time interval, we also detected a significant iron line with a centroid energy of $6.56_{-0.05}^{+0.06} \mathrm{keV}$. This is somewhat higher than the $\mathrm{Fe} \mathrm{K}_{\alpha}$ line of neutral iron $(\sim 6.4 \mathrm{keV})$, thus suggests that the X-ray flux produced by accretion onto the NS partly ionized the clump matter (according to Kallman et al. 2004, the 
centroid energy we measured at the top of the flare requires the presence of iron ions with ionization stages higher than FeXXI).

- $t=6320-9520 \mathrm{~s}$ (time intervals $\mathrm{F}, \mathrm{G}, \mathrm{H}$, and $\mathrm{I}$ ): Beginning of the flare decay. During this interval, the source X-ray flux was beginning to decrease. When it dropped below $\sim 6 \times 10^{-11}$ erg cm $\mathrm{cm}^{-2} \mathrm{~s}^{-1}$, a new rise in the $N_{\mathrm{H}}$ is measured (spectrum I). This is to be expected according to our interpretation where the X-ray flux decreases below the level required for photoionising the clump material. A further possible indication of the change in the ionization stage of the material around the NS can be deduced by the energy of the iron line detected during interval I. This is somehow lower than that measured at the top of the flare and close to the value expected in case of neutral iron (see Fig. 11).

An enigmatic finding that emerged from the analysis of the $X M M-N e w t o n$ data during this time interval is the drop in the source count-rate visible in Fig. 1 around $t=8500-9500$. As we discussed in Sect. 2.2, during this interval no particular spectral change was observed in the X-ray emission (we note only a marginal increase in the $N_{\mathrm{H}}$ with respect to the spectra extracted from the nearby time intervals and detected a significant iron line at $\sim 6.4 \mathrm{keV}$, see Table 1$)$. A closer inspection of the $X M M$-Newton lightcurve of the source around $t=8500-9500$ revealed that the apparent drop in count-rate occurred in both the low $(0.3-4 \mathrm{keV})$ and hard (4-12 keV) energy bands and that several small flares took place during this period (see Fig. 3). We suggest here that a qualitatively similar variations in the $\mathrm{X}$-ray flux from the source might be expected because of some instabilities taking place close to the NS magnetosphere (e.g., when the decreasing accretion rate of material from the clump is close to the threshold for the opening/closing of the centrifugal and/or magnetic barrier Bozzo et al. 2008a). Alternatively, it can be related to some small structures in the stellar wind located close or within the clump itself.

- $t=9520-42840 \mathrm{~s}$ (time intervals $\mathrm{J}, \mathrm{K}, \mathrm{L}, \mathrm{M}, \mathrm{N}$, and $\mathrm{O}$ ): Late stages of the flare decay. The spectral analysis revealed a progressive increase in the absorption column density, that reached a value of $N_{\mathrm{H}} \simeq 50 \times 10^{22} \mathrm{~cm}^{-2}$ in the time interval N. Even though an increase of the $N_{\mathrm{H}}$ is still expected at these times because of the lower ionizing X-ray flux, the value above is significantly higher than that measured when the source reached the peak of the X-ray flux. This result can be explained by assuming that, after the occurrence of the flare, the clump was located in front of the NS along our line of sight to the source. This would agree with the dramatic change in the X-ray spectrum of the source observed at the end of the flare during interval O ( $t=18870-42840 \mathrm{~s})$. We suggested in Sect. 2.2 that a reasonable fit to this spectrum could have been obtained with a model comprising two power-law components with different absorptions plus an iron line at $6.4 \mathrm{keV}$. This model is usually adopted to interpret the X-ray emission of sgHMXBs in eclipse (see e.g., van der Meer et al. 2005; Torrejón et al. 2010, for a review), but in the case of IGR J18410-0535, we assume that the obscuration of the NS was caused by a massive clump rather than by its supergiant companion. The more absorbed power law represents the X-ray emission caused by the accretion onto the NS that is strongly extinguished by the presence of the clump. The less absorbed power-law component is introduced to take into account the scattered emission in the stellar wind material spread all around the binary system, and might also comprise the X-ray emission produced by the shocks in the wind itself (see e.g., Bozzo et al. 2010, and references therein). This emission is unaffected by the presence of the clump and is thus only seen through an absorption column density that is compatible with the Galactic value. The iron line is caused by X-ray irradiation of cold iron in the wind of the supergiant star (see, e.g. Kallman et al. 2004), and its relatively high $E W$ also support the idea that most of the continuum emission is suppressed by the obscuration of the clump. However, if we consider that the obscuration of the $\mathrm{X}$-ray source is taking place during the entire time interval $\mathrm{O}$, some ad hoc assumptions on the system geometry would probably be required to explain how a clump with the physical dimensions estimated earlier in this section and moving with a velocity of the order of $\sim 10^{8} \mathrm{~cm} \mathrm{~s}^{-1}$ can hide the NS for about $\sim 20 \mathrm{ks}$. We suggest instead that the obscuration occurred only during the first $\sim 3 \mathrm{ks}$ of the time interval $\mathrm{O}$ (i.e. time interval $\mathrm{O}_{1}$ ). Once the clump moved away, the accretion onto the NS decreased substantially and the X-ray emission detected during the last $\sim 20 \mathrm{ks}$ of observation (time interval $\mathrm{O}_{2}$ ) was partly due to the residual accretion and partly to the scattering of these X-rays in the wind of the supergiant star and the shocks occurring within the wind itself (see e.g., Bozzo et al. 2010, and references therein). Even though the statistics of the data accumulated during the time intervals $\mathrm{O}_{1}$ and $\mathrm{O}_{2}$ was of too low quality to enable us to draw a firm conclusion, the analysis of these spectra carried out in Sect. 2 is consistent with this idea. We showed that a fit to the $\mathrm{O}_{1}$ spectrum required both the presence of the highly absorbed and the less absorbed powerlaw component, whereas the spectrum $\mathrm{O}_{2}$ could be described well using only the less absorbed power-law. However, given the very poor spectral information available during the time interval $\mathrm{O}_{2}$, we cannot presently rule out that after the clump moved away, the obscuration of the NS by its supergiant companion contributed to reduce the flux measured at this time $\left(\sim 9 \times 10^{-14} \mathrm{erg} \mathrm{cm}^{-2} \mathrm{~s}^{-1}, 1-10 \mathrm{keV}\right.$, i.e a factor of $\sim 3.5$ lower than that measured in the interval $\mathrm{A}$, see Table 1).

As a final remark, we also note that an iron line with a centroid energy and an $E W$ compatible with that measured during the time interval I and E by XMM-Newton was detected from IGR J18410-0535 during the ASCA observations carried out in 1994 and 1999 (see Sect. 1). On those occasions, the line was clearly visible in the spectra of the source extracted in the flux range $(0.1-1.0) \times 10^{-11} \mathrm{erg} \mathrm{cm}^{-2} \mathrm{~s}^{-1}(2-$ $10 \mathrm{keV}$ ), i.e. significantly different from those corresponding to the XMM-Newton detections (see Table 1). No convincing indication of the presence of an iron line could be found in the XMM-Newton spectra extracted at similar flux levels (intervals L, M, N; see also Sect. 2.2). As the centroid energy of the iron line, together with its $E W$ and normalization, provides information on the ionization state of stellar wind material and the amount of material around the NS, these results support the idea that the conditions of the stellar winds in the SFXT sources can change significantly during the NS orbits (see e.g., Walter \& Zurita Heras 2007).

\subsection{IGR J18410-0535: another SFXT hosting a slow-spinning NS?}

The timing analysis of the XMM-Newton data did not reveal any pulsations at $4.7 \mathrm{~s}$, which is the periodicity detected during previously ASCA and Swift observations of IGR J18410-0535 (see Sect. 1). The relatively tight upper limits on the pulsed 
fraction derived from the XMM-Newton data (see Table 2), convinced us to reanalyze the archival ASCA and Swift data where pulsations were detected. In the first case, our results revealed that the detection might have been due to a statistical fluctuation, while in the second case the detection was most likely related to an instrumental problem. In addition the analysis of the latest Swift observations performed during the outburst of the source that occurred on 2010 June 5 could not confirm the presence of pulsations (Romano et al. 2011). These results suggest that the NS in IGR J18410-0535 might not be pulsating at a period of $4.7 \mathrm{~s}$.

Among the $\sim 15$ sources in the SFXT class, so far only IGR J11215-5952, IGR J18483-0311, and IGR J16465-4507, displayed unambiguously coherent pulsations at $\sim 186.78 \mathrm{~s}$, $\sim 21 \mathrm{~s}$, and $\sim 228 \mathrm{~s}$, respectively (Sidoli et al. 2007; Walter \& Zurita Heras 2007; Giunta et al. 2009). However, IGR J112155952 proved to be a very peculiar source, as it is the only SFXT displaying regularly periodic outbursts connected with the NS passage at the periastron of the system, while IGR J184830311 and IGR J16465-4507 were classified as "intermediate SFXTs" due to their somewhat longer outbursts (up to a few days as opposed to a few hours) and smaller dynamic range in the Xray flux variation $\left(\lesssim 10^{3}\right.$, see e.g. Rahoui et al. 2008; Romano et al. 2010b; La Parola et al. 2010; Clark et al. 2010). All SFXTs displaying a very large dynamic range in the X-ray luminosity $\left(\gtrsim 10^{4}-10^{5}\right)$, including IGR J18410-0535, seem instead to be "non-pulsating" sources (see the cases of, e.g. XTE J1739-302 and IGR J08408-4503 and IGR J17544-2619, Bozzo et al. 2010; Rampy et al. 2009). In some of these cases, pulsations were searched deeply up to periods of several hundred seconds, and no evidence for them was found (Smith et al. 2006; Bozzo et al. 2009, 2010). These non-detections are still consistent with the possibility that these SFXT sources might host NSs with very long spin periods ( $\gtrsim 1000 \mathrm{~s})$ and that their extreme X-ray variability could be related to the centrifugal and magnetic gating mechanism (Bozzo et al. 2008a).

\section{Conclusion}

We have reported on the detection with $X M M$-Newton of a relatively bright flare from the SFXT source IGR J18410-0535. The lightcurve and spectral analysis presented in Sect. 2, provided several indications that the event was entirely due to the accretion of a massive clump onto the NS hosted in this system. Even thought the occurrence of an eclipse by the companion star toward the end of the observation could not be completely ruled out (see Sect. 5), we provided convincing evidence in favor of the "ingestion of a clump" scenario. We have shown that:

- The rise of the flare was followed by a sudden increase in the absorption column density in the direction of the source. This is expected if the event is triggered by the presence of a massive clump approaching the NS.

- At the peak of the flare, a significant drop in the absorption column density occurred; this was ascribed to the effect of the X-ray photoionization caused by the higher X-ray luminosity of the source (this is also supported by the evidence of a change in energy of the iron line centroid with the source luminosity).

- The gradual increase in the absorption column density in the direction of the source during the decay from the top of flare, together with the detection of a prominent iron line in the last part of the XMM-Newton observation, could be interpreted as implying that the remains of the clump first covered the
X-ray source for a few ks $(\sim 3 \mathrm{ks})$ and then moved away from the NS. The residual X-ray emission observed in the last $\sim 17 \mathrm{ks}$ of the observation might be due to some residual accretion taking place onto the NS.

In accordance with this interpretation, we have provided a rough estimate of the mass and radius of the clump by assuming a spherical shape. We note that, because of our poor knowledge of the properties of the binary system IGR J18410-0535 and the lack of a clear confirmation of its spin period (see Sect. 5.2), it was not possible to take into account in these calculations the effect of the NS magnetic field according to the model developed by Bozzo et al. (2008b). The lack of clear detection of relatively short spin periods in all the most extreme SFXT sources, including IGR J18410-0535, is still consistent with the possibility that these object might host NSs with very long spin periods ( $\gtrsim 1000 \mathrm{~s}$ ) and that their extreme X-ray variability could be partly related to the centrifugal and magnetic gating mechanisms (Bozzo et al. 2008a).

Acknowledgements. E.B. thanks the Swiss Society for Astronomy and Astrophysics for granting the on-going collaboration between the ISDC (Geneva, Switzerland), the ISSI (Bern, Switzerland) and the INAF-OAR (Rome, Italy). L.S. acknowledges financial support from ASI. We thank the anonymous referee for useful comments. The results presented in this paper are based on observations obtained with XMM-Newton, an ESA science mission with instruments and contributions directly funded by ESA Member States and NASA.

\section{References}

Bamba, A., Yokogawa, J., Ueno, M., Koyama, K., \& Yamauchi, S. 2001, PASJ, 53,1179

Bamba, A., Ueno, M., Koyama, K., \& Yamauchi, S. 2003, ApJ, 589, 253

Bozzo, E., Falanga, M., \& Stella, L. 2008a, ApJ, 683, 1031

Bozzo, E., Stella, L., Israel, G., Falanga, M., \& Campana, S. 2008b, MNRAS, 391, L108

Bozzo, E., Stella, L., Israel, G., Falanga, M., \& Campana, S. 2009, in AIP Conf Ser. 1126, ed. J. Rodriguez, \& P. Ferrando, 319

Bozzo, E., Stella, L., Ferrigno, C., et al. 2010, A\&A, 519, 6

Burke, B. E., Mountain, R. W., Daniels, P. J., Cooper, M. J., \& Dolat, V. S. 1993, in SPIE Conf. 2006, ed. O. H. Siegmund, 272

Cash, W. 1979, ApJ, 228, 939

Chaty, S. 2010, in ASP Conf. Ser. 422, ed. J. Martí, P. L. Luque-Escamilla, \& J. A. Combi, 243

Clark, D. J., Sguera, V., Bird, A. J., et al. 2010, MNRAS, 406, L75

de Pasquale, M., Barthelmy, S. D., Baumgartner, W. H., et al. 2010, The Astronomer's Telegram, 2661

Dickey, J. M., \& Lockman, F. J. 1990, ARA\&A, 28, 215

Ducci, L., Sidoli, L., Mereghetti, S., Paizis, A., \& Romano, P. 2009, MNRAS, 398,2152

Eversberg, T., Lepine, S., \& Moffat, A. F. J. 1998, ApJ, 494, 799

Filippova, E. V., Lutovinov, A. A., Shtykovsky, P. E., et al. 2004, Astron. Lett., 30,824

Giunta, A., Bozzo, E., Bernardini, F., et al. 2009, MNRAS, 399, 744

Grebenev, S. A., \& Sunyaev, R. A. 2007, Astron. Lett., 33, 149

Halpern, J. P., Gotthelf, E. V., Helfand, D. J., Gezari, S., \& Wegner, G. A. 2004, The Astronomer's Telegram, 289

in't Zand, J. J. M. 2005, A\&A, 441, L1

Israel, G. L., \& Stella, L. 1996, ApJ, 468, 369

Jansen, F., Lumb, D., Altieri, B., et al. 2001, A\&A, 365, L1

Kallman, T. R., Palmeri, P., Bautista, M. A., Mendoza, C., \& Krolik, J. H. 2004 ApJS, 155, 675

Krolik, J. H., \& Kallman, T. R. 1984, ApJ, 286, 366

La Parola, V., Cusumano, G., Romano, P., et al. 2010, MNRAS, 405, L66

Lépine, S., \& Moffat, A. F. J. 2008, AJ, 136, 548

Martins, F. 2011, Bull. Soc. Roy. Sci. Liège, 80, 29

Nespoli, E., Fabregat, J., \& Mennickent, R. E. 2008, A\&A, 486, 911

Ohashi, T., Ebisawa, K., Fukazawa, Y., et al. 1996, PASJ, 48, 157

Oskinova, L. M., Hamann, W., \& Feldmeier, A. 2007, A\&A, 476, 1331

Rahoui, F., Chaty, S., Lagage, P., \& Pantin, E. 2008, A\&A, 484, 801

Rampy, R. A., Smith, D. M., \& Negueruela, I. 2009, ApJ, 707, 243

Rodriguez, J., Garau, A. D., Grebenev, S., et al. 2004, The Astronomer's Telegram, 340

Romano, P., Sidoli, L., Cusumano, G., et al. 2009, MNRAS, 399, 2021 


\section{E. Bozzo et al.: A bright flare from IGR J18410-0535}

Romano, P., Cusumano, G., Baumgartner, W. H., et al. 2010a, The Astronomer's Telegram, 2662

Romano, P., Sidoli, L., Ducci, L., et al. 2010b, MNRAS, 401, 1564

Romano, P., Mangano, V., Cusumano, G., et al. 2011, MNRAS, 412, L30

Runacres, M. C., \& Owocki, S. P. 2002, A\&A, 381, 1015

Sguera, V., Bazzano, A., Bird, A. J., et al. 2006, ApJ, 646, 452

Sguera, V., Romero, G. E., Bazzano, A., et al. 2009, ApJ, 697, 1194

Sidoli, L. 2010, in AIP Conf. Ser. 1314, ed. V. Kologera, \& M. van der Sluys, 271
Sidoli, L., Romano, P., Mereghetti, S., et al. 2007, A\&A, 476, 1307 Sidoli, L., Romano, P., Mangano, V., et al. 2008, ApJ, 687, 1230

Smith, D. M., Heindl, W. A., Markwardt, C. B., et al. 2006, ApJ, 638, 974

Strüder, L., Briel, U., Dennerl, K., et al. 2001, A\&A, 365, L18

Torrejón, J. M., Schulz, N. S., Nowak, M. A., \& Kallman, T. R. 2010, ApJ, 715 947

Turner, M. J. L., Abbey, A., Arnaud, M., et al. 2001, A\&A, 365, L27

van der Meer, A., Kaper, L., di Salvo, T., et al. 2005, A\&A, 432, 999

Walter, R., \& Zurita Heras, J. 2007, A\&A, 476, 335 